OPEN ACCESS

Edited by:

Ovidiu Constantin Baltatu, Anhembi Morumbi University, Brazil

Reviewed by: Alessandro Silvani, University of Bologna, Italy Jacqueline Kathleen Phillips, Macquarie University, Australia

*Correspondence: Lisete C. Michelini michelin@usp.br

Specialty section:

This article was submitted to Integrative Physiology, a section of the journal

Frontiers in Physiology

Received: 11 September 2017 Accepted: 30 November 2017 Published: 12 December 2017

Citation:

Buttler L, Jordão MT, Fragas MG, Ruggeri A, Ceroni A and Michelini LC (2017) Maintenance of Blood-Brain

Barrier Integrity in Hypertension: A Novel Benefit of Exercise Training for Autonomic Control. Front. Physiol. 8:1048. doi: 10.3389/fphys.2017.01048

\section{Maintenance of Blood-Brain Barrier Integrity in Hypertension: A Novel Benefit of Exercise Training for Autonomic Control}

\author{
Leila Buttler, Maria T. Jordão, Matheus G. Fragas, Adriana Ruggeri, Alexandre Ceroni and \\ Lisete C. Michelini ${ }^{\star}$
}

Department Physiology and Biophysics, Institute of Biomedical Sciences, University of São Paulo, São Paulo, Brazil

The blood-brain barrier (BBB) is a complex multicellular structure acting as selective barrier controlling the transport of substances between these compartments. Accumulating evidence has shown that chronic hypertension is accompanied by BBB dysfunction, deficient local perfusion and plasma angiotensin II (Ang II) access into the parenchyma of brain areas related to autonomic circulatory control. Knowing that spontaneously hypertensive rats (SHR) exhibit deficient autonomic control and brain Ang II hyperactivity and that exercise training is highly effective in correcting both, we hypothesized that training, by reducing Ang II content, could improve BBB function within autonomic brain areas of the SHR. After confirming the absence of BBB lesion in the pre-hypertensive SHR, but marked fluorescein isothiocyanate dextran (FITC, $10 \mathrm{kD}$ ) leakage into the brain parenchyma of the hypothalamic paraventricular nucleus (PVN), nucleus of the solitary tract, and rostral ventrolateral medulla during the established phase of hypertension, adult SHR, and age-matched WKY were submitted to a treadmill training (T) or kept sedentary (S) for 8 weeks. The robust FITC leakage within autonomic areas of the SHR-S was largely reduced and almost normalized since the 2nd week of training $\left(T_{2}\right)$. BBB leakage reduction occurred simultaneously and showed strong correlations with both decreased LF/HF ratio to the heart and reduced vasomotor sympathetic activity (power spectral analysis), these effects preceding the appearance of resting bradycardia $\left(T_{4}\right)$ and partial pressure fall $\left(T_{8}\right)$. In other groups of SHR-T simultaneously infused with icV Ang II or saline (osmotic mini-pumps connected to a lateral ventricle cannula) we proved that decreased local availability of this peptide and reduced microglia activation (IBA1 staining) are crucial mechanisms conditioning the restoration of BBB integrity. Our data also revealed that Ang II-induced BBB lesion was faster within the PVN $\left(T_{2}\right)$, suggesting the prominent role of this nucleus in driven hypertension-induced deficits. These original set of data suggest that reduced local Ang II content (and decreased activation of its downstream pathways) is an essential and early-activated mechanism to maintain BBB integrity in trained SHR and uncovers a novel beneficial effect of exercise training to improve autonomic control even in the presence of hypertension.

Keywords: spontaneously hypertensive rats, blood-brain barrier, autonomic control, exercise training, angiotensin II, microglia, hypothalamus, brain stem 


\section{INTRODUCTION}

Chronic hypertension is a major risk factor for coronary artery disease, ischemic and hemorrhagic stroke, congestive heart failure, peripheral vascular, and other diseases (James et al., 2014). Accumulating experimental and clinical evidence has shown that neurohormonal control of the circulation is impaired in hypertensive individuals. Circulatory control in hypertension is characterized by baroreflex dysfunction and autonomic dysregulation, leading to sympathetic overactivity, activation of plasma and tissue renin-angiotensin system (RAS) and of circulating catecholamines. Increased angiotensin II (Ang II) availability activates intracellular pathways and increases neuronal activity within the autonomic areas as the hypothalamic paraventricular nucleus (PVN) and brainstem nuclei (nucleus of the solitary tract, NTS; rostral ventrolateral medulla, RVLM) that have been shown to contribute to neurogenic hypertension (Kang et al., 2009; Shi et al., 2010; Waki et al., 2011; Zubcevic et al., 2011). Indeed, both circulating Ang II (via $\mathrm{AT}_{1}$ receptors in endothelial cells) and locally synthetized Ang II cause vascular dysfunction and microglia activation, increase the production of reactive oxygen species, eNOS uncoupling and augment proinflammatory cytokines synthesis, which are important causal factors for sympathoexcitation in neurogenic hypertension (Shi et al., 2010; Waki et al., 2011; Zubcevic et al., 2011).

Recent studies have suggested another mechanism by which circulating Ang II gains access to autonomic areas inside the blood-brain barrier (BBB). An increased BBB permeability allowing the entry of circulating Ang II within the brain parenchyma of the PVN, RVLM, and NTS was documented in renal and spontaneously hypertensive rats (SHR) (Biancardi et al., 2014; Biancardi and Stern, 2016). Within the PVN Ang II interacts with AT1 and Toll-like receptor 4, activates microglial cells, increases local oxidative stress and causes sympathoexcitation (Biancardi et al., 2016). BBB disruption not only facilitates Ang II access, but also allows circulating inflammatory cells to enter into the brain parenchyma, contributing to further microglial activation and inflammation in autonomic areas (Waki et al., 2011; Zubcevic et al., 2011). These responses alter neurovascular coupling, dysregulate cerebral perfusion and markedly augment neuronal discharge, thus exacerbating the sympatho-humoral activation in hypertensive animals (de Vries et al., 1997; Zhang et al., 2010). Loss of BBB integrity within the cortex and hippocampus with dysregulation of local perfusion was also observed in neurodegenerative diseases including Parkinson and Alzheimer, stroke, traumatic brain injury, and other disorders (Rosenberg, 2012; Erickson and Banks, 2013; Schoknecht et al., 2015).

The search for therapies to improve BBB integrity and ameliorate the autonomic control of the circulation has been pursued by several researchers and physicians. The reduction of BBB permeability within the PVN, RVLM, and NTS of hypertensive rats treated with $\mathrm{AT}_{1}$ receptors blockade (Biancardi et al., 2014; Biancardi and Stern, 2016) highlights the importance of RAS inhibition. Besides the autonomic areas, blockade of $\mathrm{AT}_{1}$ receptors has also shown to reduce $\mathrm{BBB}$ permeability in the hippocampus and cortex (Kucuk et al., 2002; Pelisch et al.,
2011). Although $\mathrm{AT}_{1}$ receptor blockade was accompanied by neuroprotective effects in cognitive disorders (Pelisch et al., 2011) and blood pressure reduction (Kucuk et al., 2002; Biancardi et al., 2014), there is no information on autonomic control following normalization of $\mathrm{BBB}$ integrity. Moreover, it was reported recently that candesartan and ursodeoxycholic acid reduced blood pressure in obese mice but did not prevent $\mathrm{BBB}$ dysfunction and cognitive deficits (Mamo et al., 2017).

On the other hand, studies from our and other laboratories have reported the efficacy of aerobic training to downregulate brain RAS and correct autonomic dysfunction in SHR (Pan et al., 2007; Agarwal et al., 2011). Trained SHR exhibited an early (2 weeks) and maintained normalization of angiotensinogen expression within autonomic areas, which was correlated to the reduced sympathetic outflow to heart and vessels and preceded the partial pressure fall (Chaar et al., 2015). Trained SHR also showed prompt (2 weeks) normalization of baroreceptor reflex control of heart rate that coincided with a marked reduction of oxidative stress and inflammation in the PVN (Masson et al., 2014). Similar training-induced effects were also observed in other autonomic areas (Pan et al., 2007; Agarwal et al., 2011). Knowing that hypertension is characterized by autonomic impairment, BBB leakage, and Ang II-induced neuronal activation and that exercise training is highly effective to prevent Ang II-induced effects and to improve autonomic control, we hypothesized that training may improve autonomic control by restoring $\mathrm{BBB}$ integrity and normalizing brain perfusion in hypertensive individuals. Because there is no information on possible beneficial effects of exercise training on BBB leakage in the SHR (the best model of essential hypertension), we sought to evaluate in autonomic brain areas: (i) the changes on both $\mathrm{BBB}$ permeability and circulatory autonomic control induced by training starting in the chronic phase of hypertension; (ii) the combined effect of both training and elevated brain Ang II content on BBB permeability. In addition, because the BBB is completely formed and functionally competent at birth (Hagan and Ben-Zvi, 2015) we undertook a temporal study using sedentary SHR, examining the integrity of the $\mathrm{BBB}$ over time (from 1 to 5 months) to determine when the integrity of the BBB within autonomic areas becomes impaired. Normotensive Wistar Kyoto rats (WKY) were used as controls.

\section{MATERIALS AND METHODS}

\section{Animal Surgeries, Experimental Protocols, and Hemodynamic Recordings}

This study was carried out in compliance with the Ethical Principles in Animal Research of the Brazilian College of Animal Experimentation. The protocols were approved by the Institutional Animal Care and Use Committee of the University of Sao Paulo. Groups of young (20-22 days) and adult (12-13 weeks) male SHR and age-matched WKY were housed in the Animal Facilities of the Department of Physiology and Biophysics under controlled temperature/humidity, 12/12 h light/dark cycle, with free access to water and standard laboratory chow. 
SHR and age-matched WKY were submitted to three experimental protocols: (i) Blood pressure measurement and quantification of BBB permeability in autonomic areas of young rats during 5 months life span from the pre-hypertensive up to the chronic phase of hypertension; (ii) Analysis of the temporal effects induced by 8 -weeks aerobic exercise training or sedentary protocol on both hemodynamic parameters and BBB permeability in brain areas of adult SHR and WKY aged 3 month; (iii) Analysis of the combined effect of training and icv Ang II infusion on $\mathrm{BBB}$ permeability, $\mathrm{AT}_{1}$ receptor expression and microglia density within autonomic areas of the adult SHR. In all these protocols, BBB permeability was examined within the PVN, NTS, and RVLM, important autonomic nuclei controlling sympathetic and parasympathetic outflow to heart and vessels.

\section{Protocol I}

Young SHR and WKY (50-70 g) were kept sedentary in Plexiglass cages. At the 1st, 2nd, 3rd, 4th, and 5th months chronic catheters (Tygon Tubing, Critchley, Australia: $2 \mathrm{~cm}$ of 0.28:0.61 connected to $7 \mathrm{~cm}$ of $0.50: 1.50$ ID:OD) were implanted in the left femoral and left carotid arteries under anesthesia (ketamine, $80 \mathrm{mg} / \mathrm{kg}$ plus xylazine, $12 \mathrm{mg} / \mathrm{kg}$, ip). They were treated $s c$ with analgesic (ketoprofen 1\%, $2 \mathrm{mg} / \mathrm{kg}$; Biofarm, Jaboticabal, Brazil) and enrofloxacin (Baytril $5 \mathrm{mg} / \mathrm{kg}$, Bayer Sao Paulo, Brazil) and allowed to recover for $24 \mathrm{~h}$. Time-course changes of resting arterial pressure (AP) were recorded for 30-40 min (computer, $2,000 \mathrm{~Hz}$ of sampling frequency, LabChart Pro, ADInstruments Bella Vista, NSW, Australia) on the next day in conscious unrestrained rats, as previously described (Masson et al., 2014; Ichige et al., 2016). At the 1st, 3rd, and 5th month after AP recordings, $10 \mathrm{kDa}$ fluorescein isothiocyanate dextran (FITC, $10 \mathrm{mg} / \mathrm{mL}$, Sigma Aldrich, USA) plus $70 \mathrm{kDa}$ rhodamine B isothiocyanate dextran (RHO, $10 \mathrm{mg} / \mathrm{mL}$ Sigma Aldrich, USA), $286 \mu \mathrm{L} / 100 \mathrm{~g}$ each, were administered at a slow rate (70 and $300 \mu \mathrm{L} / \mathrm{min}$ in rats aged 1 and 3-5 months, respectively) via the carotid artery and allowed to recirculate for $20 \mathrm{~min}$ (Biancardi et al., 2014). Rats were then deeply anesthetized (overdose of ketamine + xylazine, $240 \mathrm{mg} / \mathrm{kg}+36 \mathrm{mg} / \mathrm{kg}$, ip) for brain harvesting immediately after the respiratory arrest. Brains were post-fixed in $4 \%$ phosphate-buffered (PB) paraformaldehyde for $48 \mathrm{~h}$, cryoprotected ( $30 \%$ sucrose in $0.01 \mathrm{M}$ PBS) for $72 \mathrm{~h}$ at $4^{\circ} \mathrm{C}$ and stored $\left(-80^{\circ} \mathrm{C}\right)$ until processing.

\section{Protocol II}

During a 2-week acclimatization period, adult SHR and WKY (250-280 g) were preselected for their ability to walk/run on a treadmill (KT-300, Inbramed, Porto Alegre, Brazil; 10 sessions from 0.4 to $0.8 \mathrm{~km} / \mathrm{h}, 0 \%$ grade, $10 \mathrm{~min} /$ day). Only active rats (those able to walk/run on the treadmill during the adaptation period, corresponding to $98 \%$ of the SHR and $93 \%$ of the WKY) were included in this protocol. Rats were then subjected to progressive maximal exercise tests (MET, Cavalleri et al., 2011) to determine maximal individual aerobic capacities, whose values were used to allocate rats with identical capacities to trained (T) and sedentary (S) groups and to set the intensity of aerobic exercise training (50-60\% of the maximal exercise capacity, performed 5 days/week, $1 \mathrm{~h}$ /day for 2 months, Masson et al.,
2014). MET were repeated at weeks 4 and 8 of both protocols in order to adjust and maintain the training intensity and compare the protocols' efficacy, respectively. Rats from S groups were handled every day and subject once per week to a short period of mild exercise (5-10 $\mathrm{min}, 0.4-0.8 \mathrm{~km} / \mathrm{h}, 0 \%$ grade) to approximate their conditions to those experienced by $\mathrm{T}$ groups (Cavalleri et al., 2011; Masson et al., 2014). At predetermined periods (weeks $0,1,2,4$, and 8 of T and S protocols), SHR and WKY were anesthetized (ketamine + xylazine, $80 \mathrm{mg} / \mathrm{kg}+12 \mathrm{mg} / \mathrm{kg}$, ip) for chronic catheterization of the femoral (hemodynamic parameters' recordings) and carotid (FITC + RHO injection) arteries. Similar to young groups, rats were treated with analgesic plus antibiotic $s c$ and recovered for $24 \mathrm{~h}$. Baseline AP and heart rate (HR) were continuously acquired on a beat-to-beat basis (30-40 min, LabChart Pro, AD Instruments, sampling frequency of $2,000 \mathrm{~Hz}$ ) on the next day in conscious freely moving rats resting in their experimental cage (Masson et al., 2014; Ichige et al., 2016). BBB permeability was analyzed after the hemodynamic recordings. The mixture of dyes was administered via the carotid artery and the rats were deeply anesthetized $20 \mathrm{~min}$ later (Biancardi et al., 2014); brains were harvested immediately after the respiratory arrest, post-fixed, cryoprotected, and stored until processing.

\section{Protocol III}

Active adult SHRs were adapted to the treadmill for 2 weeks and submitted to MET. Rats were anesthetized (ketamine, 100 $\mathrm{mg} / \mathrm{kg}+$ xylazine, $20 \mathrm{mg} / \mathrm{kg}$, ip) for the implantation of an infusion kit attached to an osmotic mini-pump within the lateral ventricle (Brain infusion kit $2+$ Micro-osmotic pump model 1002, Alzet, Cupertino CA, USA) as previously described (DeVos and Miller, 2013). Briefly, rats were placed in a stereotaxic apparatus (David Kopf, Tujunga CA, USA, incisive bar $-3.3 \mathrm{~mm}$ ) and after local anesthesia (2\% lidocaine chloridrate $+0,04 \%$ phenylephrine chloridrate) and asepsis (iodate alcohol solution), the skull was exposed and a small hole opened for the insertion of the infusion kit into the lateral ventricle (1.0 caudal to the Bregma, $1.6 \mathrm{~mm}$ lateral to the midline, $4.5 \mathrm{~mm}$ ventral to the skull surface) (Paxinos and Watson, 2007). The infusion kit was fixed with fast polymerizing methacrylate and connected to the osmotic mini-pump (0.25 $\mu \mathrm{L} / \mathrm{h}$ for 14 days) previously filled with saline or angiotensin II (Ang II, $50 \mathrm{ng} / \mu \mathrm{L} / \mathrm{h}$ ) and inserted subcutaneously in the scapular region. The skin was sutured; the rats were treated with analgesic plus antibiotic and allowed to recover in their individual cages. Exercise training $(\mathrm{T}=50-$ $60 \%$ of maximal exercise capacity, $1 \mathrm{~h} /$ day) or the $\mathrm{S}$ protocol started in the next day and continued for another 13 days, during the active infusion period of the mini-pumps. Notice that 14 consecutive daily exercise sessions and training for 2-3 weeks (protocol II) represent a similar volume of training, that is, they are isocaloric. At the end of protocols rats were anesthetized and cannulated. In the following day, after hemodynamic recordings half of these rats were infused with FITC $+\mathrm{RHO}$ intra-arterially and brains removed $20 \mathrm{~min}$ later for immunofluorescence assays, as described above; in the other half, the brains were quickly perfused with sterile $0.01 \mathrm{M}$ PBS $(100 \mathrm{~mL}, \mathrm{pH}$ 7.4) followed by $4 \%$ paraformaldeyde $(400 \mathrm{~mL}, 20-30 \mathrm{~mL} / \mathrm{min}$, Daigger pump, 
Vernon Hills IL, USA). Brains were removed, post-fixed (4h), cryoprotected $\left(72 \mathrm{~h}\right.$ at $\left.4^{\circ} \mathrm{C}\right)$, stored $\left(-80^{\circ} \mathrm{C}\right)$ until processing and used to analyze the combined effects of training and Ang II infusion on AT1 receptors' expression and microglia activation.

\section{Power Spectral Analysis}

Time series of resting systolic AP (SAP) and pulse interval (PI) were used to analyze pressure and HR variability, as previously described (Japundzic et al., 1990; Task Force of the European Society of Cardiology, North American Society of Pacing and Electrophysiology, 1996; Eckberg, 1997). Briefly, time- and frequency-domain analyses were evaluated using fast Fourier transformation by Welch's method and Hanning windows with $50 \%$ overlap. Power spectral density for low frequency (LF, 0.20 $0.75 \mathrm{~Hz}$, indicating mainly the sympathetic activity to vessels and sympathetic + parasympathetic modulation of the heart) and high frequency domain ( $\mathrm{HF}, 0.75-3.0 \mathrm{~Hz}$, indicative of the vagal activity to the heart) were obtained by means of power spectrum density integration within each frequency bandwidth, using a customized routine (MATLAB6.0, Mathworks, Natick, MA, USA. Ceroni et al., 2009; Masson et al., 2014). LF/HF ratio to the heart and the spontaneous baroreflex sensitivity through the $\alpha \mathrm{LF}$ and $\alpha \mathrm{HF}$ indexes (representing baroreflex sensitivity at the LF and HF bands, respectively, Laude et al., 2004; Martelli et al., 2014) were also assessed.

\section{BBB Leakage Analysis}

Sequential coronal brain slices $(30 \mu \mathrm{m})$ containing the PVN, NTS, and RVLM (Paxinos and Watson, 2007) were obtained in the cryostat (Leica CM1850, USA), collected in tissue culture wells with $0.1 \mathrm{M}$ PBS, placed on gelatinized slides and mounted with Slowfade $\AA$ Gold Antifade Reagent (Life Technologies CA, USA). Tissues were examined on a fluorescence microscope (Leica DMLB, Nussloch, Germany) attached to an ExiBlue camera (Imaging, Canada); the selected images were acquired by the Image-Pro Plus software (v7.01, Media Cybernetics, USA) and quantified by the ImageJ software (NIH, USA). BBB permeability, evaluated by the capability of the small size fluorescent dextran (FITC) to remain within vessels or partially leak into the brain parenchyma in the presence of compromised BBB integrity, was quantified as previously described (Biancardi et al., 2014). Briefly, a binary image containing only the FITC signal (extra + intravascular), was subtracted from a binary image of the colocalized RHO-FITC image (intravascular only). The degree of leaked FITC in the subtracted image was quantified by the proportion of positive pixels in the acquired image; individual results were averaged per group and means compared among experimental groups. To confirm the specificity of BBB permeability within autonomic areas, FITC leakage was also analyzed in the hypoglossus nucleus, which does not participate in autonomic control and is not affected by exercise training.

\section{Immunofluorescence Assays}

Similar coronal brain sections interesting the PVN, NTS, and RVLM were sequentially collected in four wells with $0.1 \mathrm{M}$ PBS at $4{ }^{\circ} \mathrm{C}$. Before the incubation with specific antibodies, slices were pre-treated with a solution containing $1 \%$ of hydrogen peroxide and $10 \%$ methanol diluted in $0.1 \mathrm{M}$ PBS for $30 \mathrm{~min}$ at $4^{\circ} \mathrm{C}$. After 310 -min washes in $0.1 \mathrm{M}$ PBS, the slices were preincubated in $2 \%$ normal donkey serum (S30-10ML, Millipore Temceula, CA, USA) for $1 \mathrm{~h}$. Slices were then incubated on a shaker for $48 \mathrm{~h}$ at $4{ }^{\circ} \mathrm{C}$ with the following primary antibodies: mouse anti-rat blood-brain barrier monoclonal antibody (SMI 71, 1:2,000; Covance, CA, USA); rabbit anti-angiotensin type 1 receptor (AT1 (306):sc-579, 1:50, Santa Cruz Biotechnology, CA, USA) plus mouse anti-neuronal nuclei (NeuN) (1:1,000; Millipore, Darmstadt, German); or rabbit anti-mouse IBA-1 (1:1,000; Wako, Osaka, Japan). Primary antibodies were diluted in $0.1 \mathrm{M}$ PBS containing $0.1 \%$ Triton X-100. Tissue slices were then incubated for $1 \mathrm{~h}$ at room temperature with secondary antibodies (Jackson Immunoresearch Laboratories Inc., MD, USA): Alexa Fluor 488-conjugated AffiniPure donkey anti-mouse $\operatorname{IgG}(\mathrm{H}+\mathrm{L}), 1: 500$ dilution, Alexa Fluor 594-conjugated AffiniPure donkey anti-rabbit $\operatorname{IgG}(\mathrm{H}+\mathrm{L})$, 1:500 dilution or Alexa Fluor 488-conjugated AffiniPure donkey anti-rabbit IgG $(\mathrm{H}+\mathrm{L}), 1: 500$ dilution. Tissue slices in the 4 th well were used for negative controls (absence of primary or secondary antibody). After 3 other 10 -min washes with $0.1 \mathrm{M}$ PBS, the slices were mounted

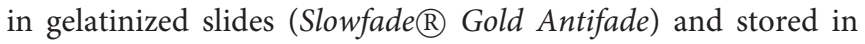
dark boxes at $4^{\circ} \mathrm{C}$. Images from all experimental groups were digitized with identical acquisition settings and analyzed (Leica DMLB) by an investigator blind to group allocation. A threshold paradigm was used for normalization and quantification of the immunofluorescence signal, as previously described (HigaTaniguchi et al., 2007). Briefly an averaged background staining was obtained from each image and only signal intensities 1.5-times higher than the calculated background fluorescence were considered for the analysis. Immunofluorescence signals (integrated density) were measured within the ventromedial, dorsal cap, and posterior autonomic subnuclei of the PVN (AOIs of $28,884,5,135,46,405 \mu \mathrm{m}^{2}$, respectively), NTS (AOI of $48,378 \mu \mathrm{m}^{2}$ ), and RVLM (AOI of $42,168 \mu \mathrm{m}^{2}$ ) and expressed in arbitrary units $/ \mu \mathrm{m}^{2}$. Quantifications were performed in both left and right side of the nuclei; values from sections were averaged to give a mean value for the nucleus for each rat in each condition.

\section{Statistical Analysis}

Data are presented as mean \pm SEM. MAP and BBB leakage changes in WKY and SHR groups during life span and treadmill performance during $\mathrm{S}$ and $\mathrm{T}$ conditions were analyzed by twoand three-way ANOVA with repeated measurements (time), respectively. Training effects in SHR-T and WKY-T during the 8 -weeks protocol were analyzed by the factorial ANOVA. The comparison of functional data and BBB leakage area between groups (SHR-S, SHR-T, WKY-S, and WKY-T) at different time points (weeks 0 and 8) was made by two-way ANOVA. Comparison of BBB leakage in rats infused with saline or Ang II was analyzed by one-way ANOVA. Unpaired $t$-test as used to compare hypoglossus BBB leakage area between SHR and WKY groups. Fisher was used as the post-hoc test. Correlation analyses were performed using Pearson's statistics. Bonferroni correction was used to ratify the significance level for the different correlations made. All analyses were performed using 
the STATISTICA 12.0 (Vince Stat Software Inc). Differences were considered significant at $P<0.05$.

\section{RESULTS}

\section{Blood Pressure and BBB Permeability Changes during Development in SHR and WKY}

Our first approach was to analyze time-course changes on BBB permeability during life span since the pre-hypertensive phase. Resting mean AP (MAP) was measured in conscious sedentary SHR and WKY aged 1, 2, 3, 4, and 5 months (Figure 1A) and the integrity of $\mathrm{BBB}$ in autonomic brain areas was evaluated at the 1 st, 3rd, and 5th month (Figures 1B,C). There was no significant BBB leakage in WKY and SHR aged 1 month. In the sedentary WKY, 5-months life span was accompanied by very small although significant increases in BBB leakage $(0.7$ $\pm 0.1,0.7 \pm 0.1$, and $0.5 \pm 0.1 \%$ in the PVN, NTS, and RVLM, respectively, at the 5 th vs. 1st month, Figure 1C). In contrast, the establishment of hypertension was accompanied by marked increases in FITC extravasation within the brain parenchyma $(P<0.001$ for group, age and interaction in the three areas analyzed): in SHR aged 3 months, dye leakage was $7.2 \pm 1.3,11.5 \pm 1.3$, and $5.8 \pm 0.8 \%$ within the PVN, NTS, and RVLM, respectively (Figure 1C), corresponding to increases over 100 times, when compared to age-matched WKY. From the early to a late phase of hypertension, leakage area was unaltered in the NTS and RVML, but it increased in the PVN (12.4 $\pm 0.9 \%$ at the 5 th month). Interestingly, hypertensioninduced $\mathrm{BBB}$ disruption was not observed in the hypoglossus nucleus, since FITC extravasation was small and similar in both sedentary strains aged 3 months (WKY $=0.23 \pm 0.05 \%$; SHR $=0.24 \pm 0.06 \%$ ).

\section{Temporal Effects Induced by Training on Hemodynamic Parameters and Their Variabilities in Adult SHR and WKY}

SHR and WKY aged 3 months were submitted to training (T) or sedentary (S) protocols. Besides the better treadmill performance exhibited by the SHR since the beginning of protocols, both $\mathrm{T}$ groups showed proportional increases in attained velocities and similar performance gain at the end of protocols $(+73 \%$ for SHR$\mathrm{T}$ and WKY-T vs. respective values at week $0, P=0.003$ for time, $P<0.001$ for group, condition and interaction effects, Table 1). The performance gain was not changed in WKY-S, but significantly decreased in SHR-S ( $-40 \%$, Table 1$)$. Both groups exhibited similar body weight at week 0. Except for the SHR-S that exhibited a slight higher value, body weight gain during $\mathrm{T}$ and $S$ protocols was similar in the other groups (Table 1).

SHR had already attained the established phase of hypertension at the beginning of $\mathrm{T}$ and $\mathrm{S}$ protocols (average increases of 23 and $11 \%$ in resting MAP and $\mathrm{HR}$, vs. respective values in WKY, Table 2). Training affected differentially resting values of MAP $(P<0.001$ for group and condition; time, $P=0.045$; interaction, $P=0.269)$ and $\operatorname{HR}(P<0.001$ for group, condition and time; $P=0.278$ for interaction). SHR-T exhibited significant MAP fall $(-13 \%)$ and resting bradycardia $(-13 \%)$ from $\mathrm{T}_{4}$ up to $\mathrm{T}_{8}$, while age-matched WKY-T only showed resting bradycardia $\left(-12 \%\right.$ from $\mathrm{T}_{1}$ on, Table 2$)$ when compared to respective sedentary controls. Although AP and HR values were not changed in $\mathrm{S}$ groups, $\mathrm{SHR}-\mathrm{S}_{8}$ showed a 2.2 -fold increase in SAP variability when compared to SHR- $S_{0}(P<0.001$ for group and condition; $P=0.122$ for time; $P=0.006$ for interaction, Table 3$)$. At the end of protocols, $S H R-S_{8}$ vs. WKY$\mathrm{S}_{8}$ exhibited higher sympathetic vasomotor modulation (LF-SAP increased by 5.1 -fold, Table 3 ). These effects were completely abrogated by exercise training, with a significant reduction of LF-SAP $(-45 \%)$ since $\mathrm{T}_{2}(P<0.001$ for group and condition; $P=0.035$ for time; $P=0.241$ for interaction). Pulse interval (PI) variability was almost similar in sedentary groups, unaffected during the 8 -weeks sedentary protocol, but augmented in both trained strains $(P=0.115$ for group; $P=0.017$ for condition; $P<0.001$ for time; $P=0.505$ for interaction): PI variability was significantly increased in SHR-T T $_{4}$ and SHR-T 8 (2.8- and 4.4-fold increases, respectively) and in $\mathrm{WKY}_{4}$ (2.5-fold, Table 3 ). Although similar increases in PI variability ( $\sim 2.0$-fold change) were observed in WKY at the 1st, 2nd, and 8th weeks of training, the large individual variabilities precluded significant changes. Interestingly, training-induced increase on PI variability was caused by different mechanisms in each strain (Table 3 ): an early and maintained $\left(\mathrm{T}_{2}\right.$ and $\mathrm{T}_{8}$ ) elevation of HF-PI in the SHR and a late $\left(\mathrm{T}_{8}\right)$ reduction of LF-PI in the WKY. Spontaneous baroreflex sensitivity (as indicated by $\alpha \mathrm{LF}$ and $\alpha \mathrm{HF}$ indexes in Table 3 ), was depressed in the SHRs vs. WKYs and improved by exercise training in both groups $(P<0.001$ for group and condition; $P=0.045$ for time; $P=0.723$ for interaction effect). These training-induced changes in autonomic modulation significantly reduced the LF/HF ratio in both trained groups.

\section{Temporal Effects Induced by Training on BBB Permeability in Adult SHR and WKY}

As documented in Figure 1, SHR aged 3 months exhibited marked FITC leakage within the brain parenchyma of the three autonomic areas. Photomicrographs and quantitative PVN data obtained during $\mathrm{S}$ and $\mathrm{T}$ protocols show that $(P<0.001$ for group, condition, time, and interaction): (i) the leakage area was significantly increased in SHR kept sedentary for 8 weeks $(+72 \%$ from $\mathrm{S}_{0}$ to $\mathrm{S}_{8}$, Figures 2A,B); (ii) training caused a prompt and marked improvement on $\mathrm{BBB}$ integrity in the PVN from $\mathrm{T}_{2}$ on ( $-79 \%$, Figure 2B); (iii) BBB leakage area was reduced by $91 \%$

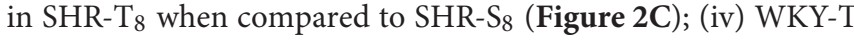
also showed a small improvement on BBB integrity (FITC leakage was reduced by $28 \%$, Figure 2C).

Except for the maintained leakage during the 8-weeks $\mathrm{S}$ protocol, similar patterns were observed in the NTS and RVLM of the SHR group (illustrative images were not shown). Quantitative data $(P<0.001$ for group and condition; $P=0.501$ for time; $P=0.023$ for interaction) confirmed that 2 weeks of training significantly reduced both NTS $(-89 \%, P<0.05$, Figure 2D) and RVLM leakage area $(-83 \%, P<0.05$, Figure $2 \mathrm{~F})$, values that were maintained up to the end of protocols. Exercise training also caused small reduction on FITC leakage within the NTS 

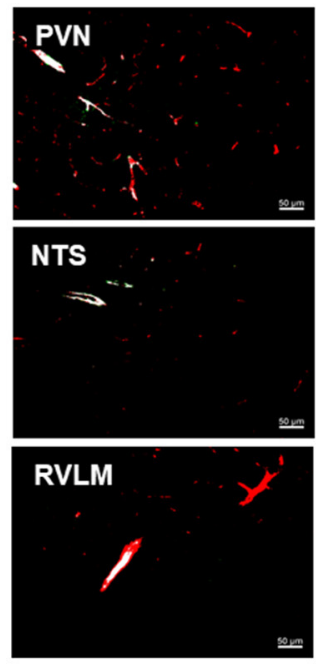

1 month
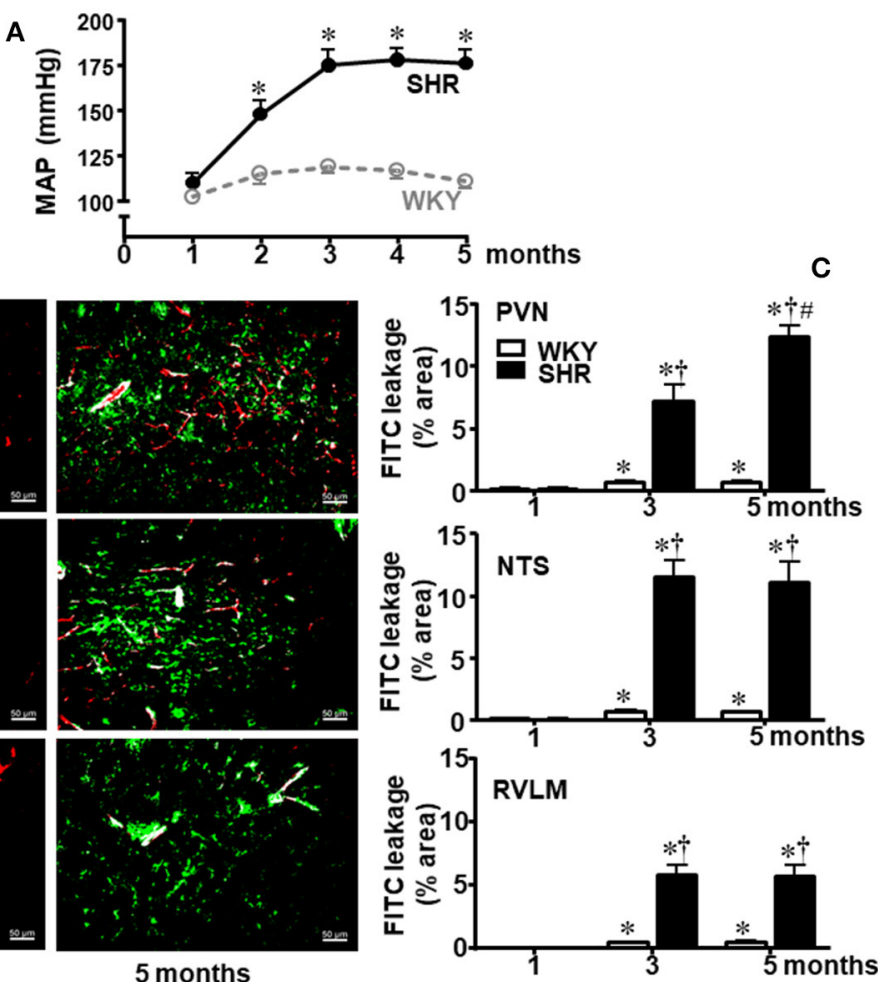

FIGURE 1 | Temporal changes of blood-brain barrier permeability within autonomic areas during 5 months' life span in sedentary spontaneously hypertensive (SHR) and normotensive rats (WKY). (A) Resting mean arterial pressure (MAP) values in SHR and WKY during 5 months' life span. $n=8$ rats/subgroup. Significances $\left({ }^{\star} P<0.05\right)$ vs. WKY. (B) Images show the capillary profile (red, Rhodamine) and FITC extravasation (green) into the brain parenchyma in the paraventricular nucleus of the hypothalamus (PVN), nucleus of the tract solitary (NTS), and rostral ventrolateral medulla (RVLM) in SHR aged 1 and 5 months. (C) Graphs compare FTIC leakage changes within the three autonomic areas of SHR and WKY groups from the 1st up to the 5th month. Values are the means of 8-12 slices, 4 rats in each subgroup.

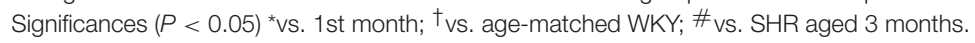

TABLE 1 | Treadmill performance and body weight of WKY and SHR groups during training $(\mathrm{T})$ or sedentary $(\mathrm{S})$ protocols.

\begin{tabular}{|c|c|c|c|c|}
\hline & WKY-S & WKY-T & SHR-S & SHR-T \\
\hline \multicolumn{5}{|c|}{ TREADMILL PERFORMANCE DURING S AND T PROTOCOLS } \\
\hline week 0 (km/h) & \multicolumn{2}{|c|}{$\begin{array}{c}1.1 \pm 0.1 \\
(n=42)\end{array}$} & \multicolumn{2}{|c|}{$\begin{array}{c}1.5 \pm 0.2^{*} \\
(n=74)\end{array}$} \\
\hline week 4 (km/h) & $\begin{array}{c}1.2 \pm 0.1 \\
(n=8)\end{array}$ & $\begin{array}{c}1.7 \pm 0.1^{\# \dagger} \\
(n=16)\end{array}$ & $\begin{array}{c}1.1 \pm 0.2^{\#} \\
(n=12)\end{array}$ & $\begin{array}{c}2.3 \pm 0.1^{\#+*} \\
(n=34)\end{array}$ \\
\hline week 8 (km/h) & $\begin{array}{c}1.0 \pm 0.0 \\
(n=8)\end{array}$ & $\begin{array}{c}1.9 \pm 0.2^{\# \dagger} \\
(n=8)\end{array}$ & $\begin{array}{c}0.9 \pm 0.1^{\#} \\
(n=12)\end{array}$ & $\begin{array}{c}2.6 \pm 0.2^{\# \dagger \star} \\
(n=14)\end{array}$ \\
\hline gain (\% change) & -8 & +73 & -40 & +73 \\
\hline \multicolumn{5}{|c|}{ BODY WEIGHT DURING S AND T PROTOCOLS } \\
\hline week 0 (g) & \multicolumn{2}{|c|}{$\begin{array}{l}265 \pm 5 \\
(n=42)\end{array}$} & \multicolumn{2}{|c|}{$\begin{array}{l}261 \pm 2 \\
(n=74)\end{array}$} \\
\hline week 4 (g) & $\begin{array}{c}298 \pm 9^{\#} \\
(n=8)\end{array}$ & $\begin{array}{c}303 \pm 9^{\#} \\
(n=16)\end{array}$ & $\begin{array}{c}303 \pm 5^{\#} \\
(n=12)\end{array}$ & $\begin{array}{c}294 \pm 4^{\#} \\
(n=34)\end{array}$ \\
\hline week 8 (g) & $\begin{array}{c}304 \pm 8^{\#} \\
(n=8)\end{array}$ & $\begin{array}{c}311 \pm 9^{\#} \\
(n=8)\end{array}$ & $\begin{array}{c}329 \pm 5^{\#} \\
(n=12)\end{array}$ & $\begin{array}{c}305 \pm 5^{\# \dagger} \\
(n=14)\end{array}$ \\
\hline BW gain $(g)$ & $+42 \pm 7$ & $+45 \pm 6$ & $+65 \pm 5^{\star}$ & $+43 \pm 4^{\dagger}$ \\
\hline
\end{tabular}

Values are means \pm SEMs. Gain represents the difference between experimental weeks 8 and 0 . The number of rats is indicated in parenthesis. Significances $(P<0.05)$ are ${ }^{*} v s$. WKY; ${ }^{*}$ vs. week $0 ;{ }^{\dagger}$ vs. respective $S$ group. and RVLM of the WKY group ( -24 and $-11 \%$, Figures 2E,G, respectively).

\section{Training-Induced Improvement of BBB Integrity Correlates with the Amelioration of Autonomic Function}

Since the preservation of BBB integrity in autonomic nuclei of trained rats and the improvement and/or correction of autonomic modulation exhibit similar time course changes, we evaluate whether these parameters are correlated. Traininginduced reduction of the PVN leakage area was positively correlated with the decrease of both LF-SAP (an index of sympathetic vasomotor outflow) and SAP variability only in the SHR group (Figure 3; correlation coefficients and $\mathrm{P}$ values are presented in Table 4). Reduced PVN leakage area was positively correlated with decreased LF/HF ratio to the heart and negatively correlated with spontaneous baroreflex sensitivity in the SHR group (Figure 3, Table 4). On the other hand, reduction of PVN leakage area was negatively correlated with increased PI variability in both SHR and WKY groups. Similar correlations between improvement of autonomic functions and improvement of BBB integrity were observed for the NTS and RVLM in the hypertensive strain (Table 4). 
Combined Effects of Training and icv Ang II Infusion on BBB Permeability in Adult SHR

Because main changes on BBB permeability were observed in the hypertensive strain and knowing that high Ang II availability increases (Biancardi et al., 2014) while exercise training decreases BBB leakage (present set of data), next we analyzed in the

TABLE 2 | Time course changes of resting arterial pressure (AP) and heart rate $(\mathrm{HR})$ in sedentary (S) and trained (T) WKY and SHR groups during the 8 experimental weeks.

\begin{tabular}{|c|c|c|c|c|}
\hline Groups & SAP $(\mathrm{mmHg})$ & DAP $(\mathrm{mmHg})$ & MAP (mmHg) & $\mathrm{HR}(\mathrm{b} / \mathrm{min})$ \\
\hline \multicolumn{5}{|l|}{ WKY } \\
\hline $\mathrm{S}_{0}=\mathrm{T}_{0}$ & $146 \pm 4$ & $113 \pm 3$ & $128 \pm 2$ & $363 \pm 18$ \\
\hline $\mathrm{T}_{1}$ & $148 \pm 5$ & $116 \pm 4$ & $128 \pm 3$ & $320 \pm 11 \#$ \\
\hline $\mathrm{T}_{2}$ & $146 \pm 6$ & $112 \pm 5$ & $126 \pm 5$ & $312 \pm 13 \#$ \\
\hline $\mathrm{T}_{4}$ & $149 \pm 8$ & $113 \pm 5$ & $127 \pm 8$ & $303 \pm 16 \#$ \\
\hline$T_{8}$ & $147 \pm 6$ & $114 \pm 4$ & $127 \pm 5$ & $321 \pm 10^{\# \dagger}$ \\
\hline $\mathrm{S}_{8}$ & $145 \pm 4$ & $110 \pm 3$ & $123 \pm 3$ & $347 \pm 15$ \\
\hline \multicolumn{5}{|l|}{ SHR } \\
\hline $\mathrm{S}_{0}=\mathrm{T}_{0}$ & $198 \pm 9^{\star}$ & $156 \pm 6^{\star}$ & $175 \pm 7^{*}$ & $404 \pm 11^{*}$ \\
\hline $\mathrm{T}_{1}$ & $186 \pm 7^{\star}$ & $145 \pm 6^{\star}$ & $161 \pm 6^{\star}$ & $383 \pm 12^{*}$ \\
\hline $\mathrm{T}_{2}$ & $179 \pm 10^{*}$ & $141 \pm 9^{*}$ & $157 \pm 10^{*}$ & $366 \pm 7^{*}$ \\
\hline $\mathrm{T}_{4}$ & $173 \pm 7^{\star}$ & $138 \pm 8^{*}$ & $153 \pm 7^{\# *}$ & $351 \pm 11^{\star \#}$ \\
\hline$T_{8}$ & $170 \pm 6^{\# \dagger *}$ & $137 \pm 5^{\#+*}$ & $153 \pm 6^{\#+*}$ & $355 \pm 5^{\star \dagger \#}$ \\
\hline $\mathrm{S}_{8}$ & $189 \pm 8^{*}$ & $148 \pm 6^{\star}$ & $176 \pm 8^{\star}$ & $392 \pm 13^{*}$ \\
\hline
\end{tabular}

Values are means $\pm S E M$; SAP, systolic AP; DAP, diastolic AP; MAP, mean AP. Hemodynamic measurements were made in 4-5 WKY/subgroup and 8-10 SHR/subgroup. Significances $(P<0.05)$ are ${ }^{*} v s$. week-matched WKY; ${ }^{\#} v s$. respective week $0{ }^{\dagger}$ vs. respective $S_{8}$.
SHR the combined effects of both. According to our previous studies (Masson et al., 2014, 2015; Chaar et al., 2015) and the present observations showing that training-induced autonomic adaptations as well as improvement of BBB integrity in the SHR occurred after 2 weeks of exercise training, we extended this protocol for 14 experimental days. Notice that 14 daily consecutive sessions of exercise correspond to the volume of training attained between experimental weeks 2 and 3 of the previous protocol in which the rats trained 5 days/week.

SHR-T simultaneously infused with $i c v$ sterile saline during 14-days showed very small FITC leakage into the brain parenchyma of the PVN, NTS, and RVLM (Figures 4A,B), a response similar to that observed previously. In contrast, SHR$\mathrm{T}$ infused with Ang II icv for 14 days exhibited significant BBB leakage within the PVN $(5.3 \pm 0.6 \%$, Figures $4 A, B)$. Interestingly, 14-days Ang II infusion caused small increases but did not significantly change FITC leakage area within the NTS and RVLM of the SHR-T. To confirm the effects of Ang II and exercise training on the lesion and/or integrity of the BBB, next we analyzed the expression of endothelial barrier antigen (EBA), a luminal protein expressed only in cerebral microvessels with intact BBB (Lin and Ginsberg, 2000; Norsted et al., 2008). When compared to SHR-S, EBA staining was significantly elevated in the three autonomic areas of the SHR-T, with a larger increase in the PVN (Figure 4C). Accordingly, simultaneous Ang II treatment blocked the effect of training.

Knowing that $\mathrm{BBB}$ disruption facilitates the access of circulating Ang II to neurons and microglial cells (Biancardi et al., 2014), next we analyzed the effects of training and Ang II treatment on the expression of $\mathrm{AT}_{1}$ receptors in neurons and on microglia activation within the PVN, NTS, and RVLM. Unexpectedly, training was accompanied by an upregulation of

TABLE 3 | Changes in systolic arterial pressure (SAP) and pulse interval (PI) variabilities and respective spectral components induced by training (T) or sedentary (S) protocols in adult SHR and WKY groups.

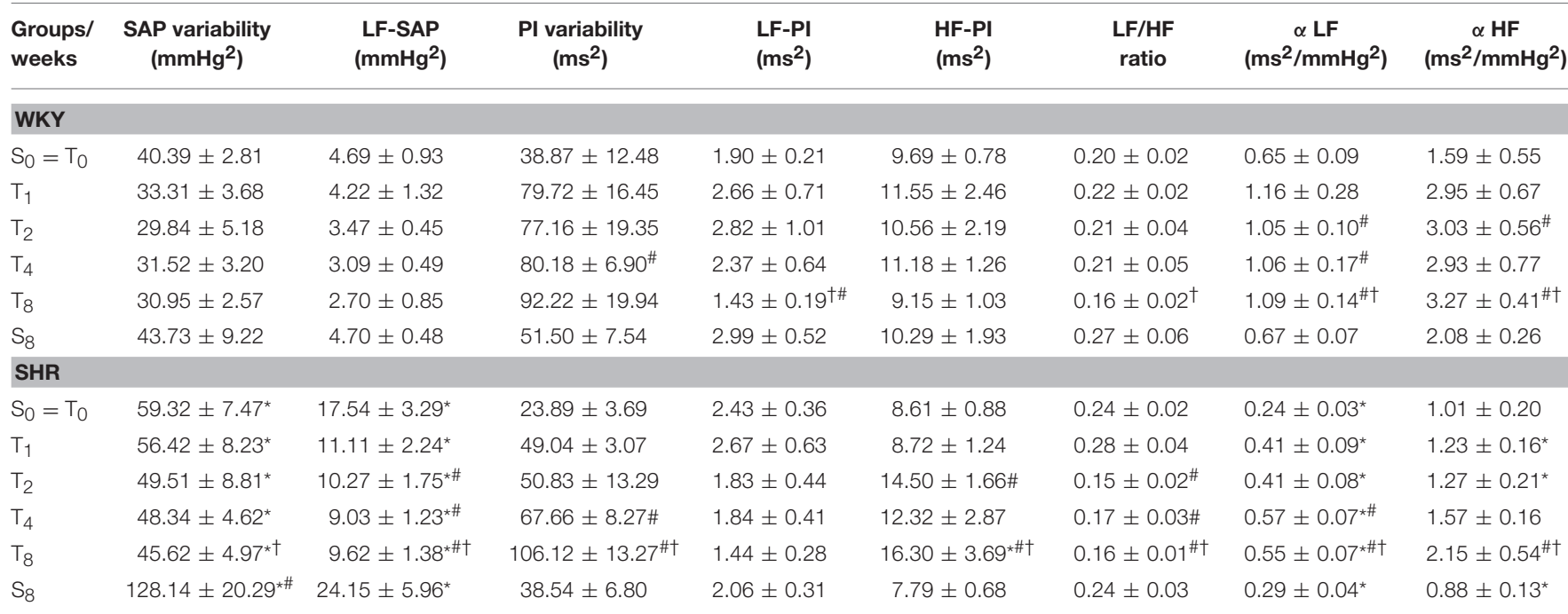

Values are means $\pm S E M S$. LF, low-frequency component; $H F$, high-frequency component. aLF and aHF represent the spontaneous baroreflex sensitivity calculated with the LF or HF components of Pl and corresponding SAP components. Measurements were made in 4-5 WKY/subgroup and 8-10 SHR/subgroup. Significances $(P<0.05)$ are ${ }^{*} v S$. week-matched WKY; $\#$ vs. respective week $0 ;{ }^{\dagger}$ vs. respective $S_{8}$. 


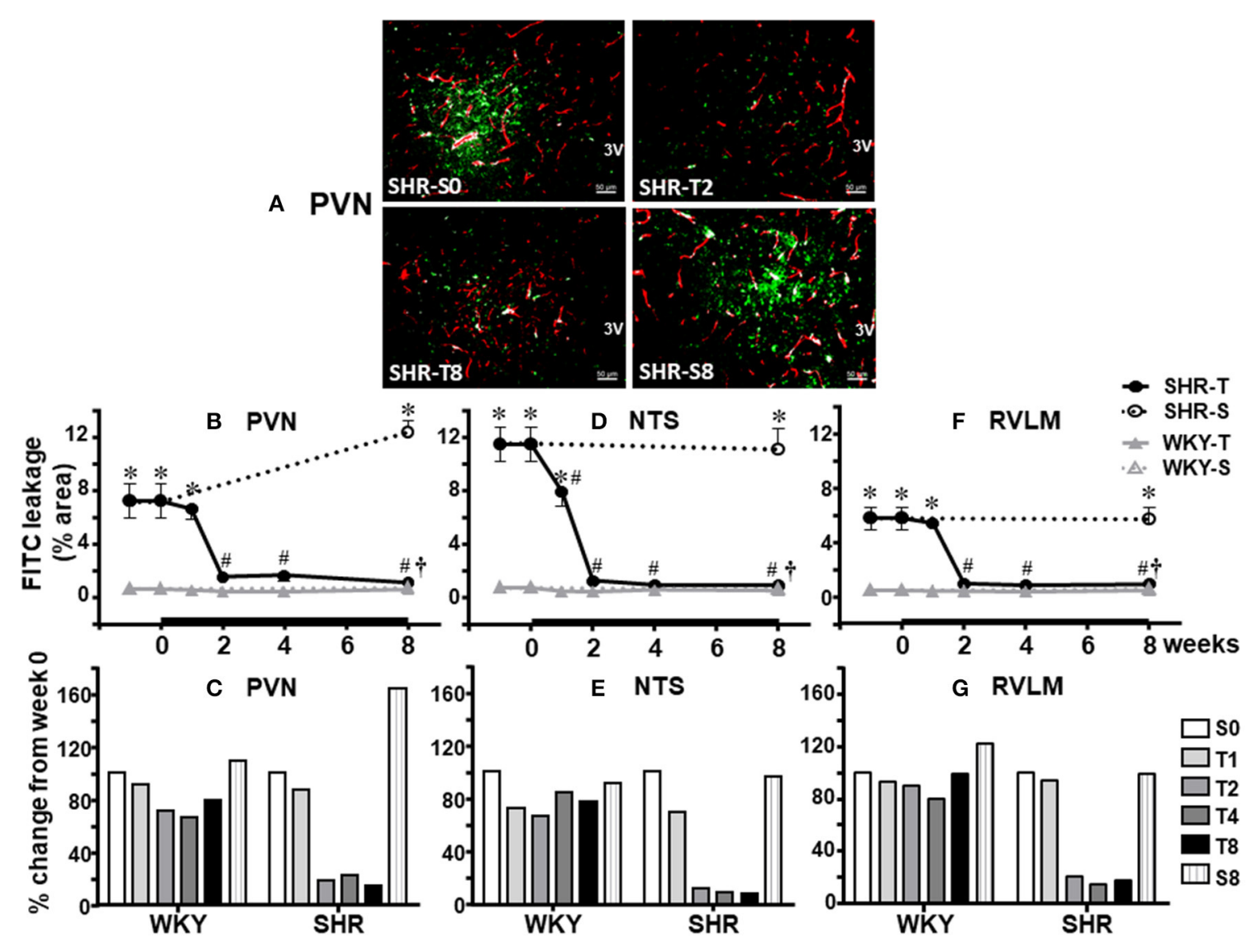

FIGURE 2 | Temporal changes of blood-brain barrier permeability induced by aerobic training in autonomic areas of spontaneously hypertensive (SHR) and normotensive rats (WKY) aged 3 months. (A) Representative image showing the capillary profile (red) and the effects of 2 and 8 weeks of exercise training on FITC extravasation (green) within the brain parenchyma of the paraventricular nucleus of the hypothalamus (PVN). For comparison images of the sedentary (S) SHR are shown at weeks 0 and 8. Graphs depict training-induced changes on absolute leakage area (B,D,F) and percent changes from values at week 0 (C,E,G) observed within the PVN $(\mathbf{B}, \mathbf{C})$, nucleus of the solitary tract (NTS, D,E) and the rostral ventrolateral medulla (RVLM, F,G) of the SHR and WKY groups. Values are the means of 8-12 slices, 4 rats in each subgroup. Significances $(P<0.05)$ are *vs. WKY; \# vs. week 0; ${ }^{\dagger}$ vs. respective $S$ groups.

$\mathrm{AT}_{1}$ receptors in $\mathrm{PVN}$ neurons, an effect that was blocked by simultaneous Ang II $i c v$. infusion (Figures 5A,B). Again, training and Ang II effects were more prominent within the PVN when compared to NTS and RVLM (quantitative data in Figure 5B; illustrative images were not shown). Photomicrographs of microglia expression in the PVN of SHR-T treated with saline (Figure 6A) show that training markedly reduced its density and kept microglial cells in an inactive state (long and ramified processes), while this response was completely blocked in SHR-T treated with Ang II that exhibited increased microglia expression and activated microglial cells (rhomboid shape, with short and thicker processes-insets in Figure 6A). Quantitative analysis ratified the attenuation of microglia activation within the PVN and NTS of the SHR-T (reduced IBA-1 expression, Figure 6B), without significant changes in the RVLM (illustrative images of IBA 1 staining in the NTS and RVLM were not shown). The blockade of training effect within the PVN by simultaneous infusion of Ang II confirmed this effect was mediated by reduced local availability of the neurohormone in the SHR-T and that Ang II effects were faster within this autonomic area.

\section{DISCUSSION}

The present set of data confirm previous observations of hypertension-induced BBB disruption in brain areas modulating the autonomic control of the circulation (Biancardi et al., 2014; Biancardi and Stern, 2016) showing in addition a new findingthe complete blockade of this deleterious effect by the exercise training, even in the presence of hypertension. There are several original observations: (i) BBB disruption is absent in the PVN, NTS, and RVLM of the SHR at the pre-hypertensive phase, but fully manifested in the established phase of hypertension; (ii) within the PVN there is a progressive increase in BBB dysfunction during the maintained phase of hypertension, an effect not observed in other autonomic nuclei; (iii) exercise training rapidly reverses dye leakage, increases the expression of endothelial barrier antigen and almost normalizes PVN, NTS, and RVLM BBB permeability, an effect strongly correlated with reduced sympathetic vasomotor activity, decreased pressure variability, and increased heart rate variability observed in the trained SHR; (iv) training-induced reduction of brain Ang II 

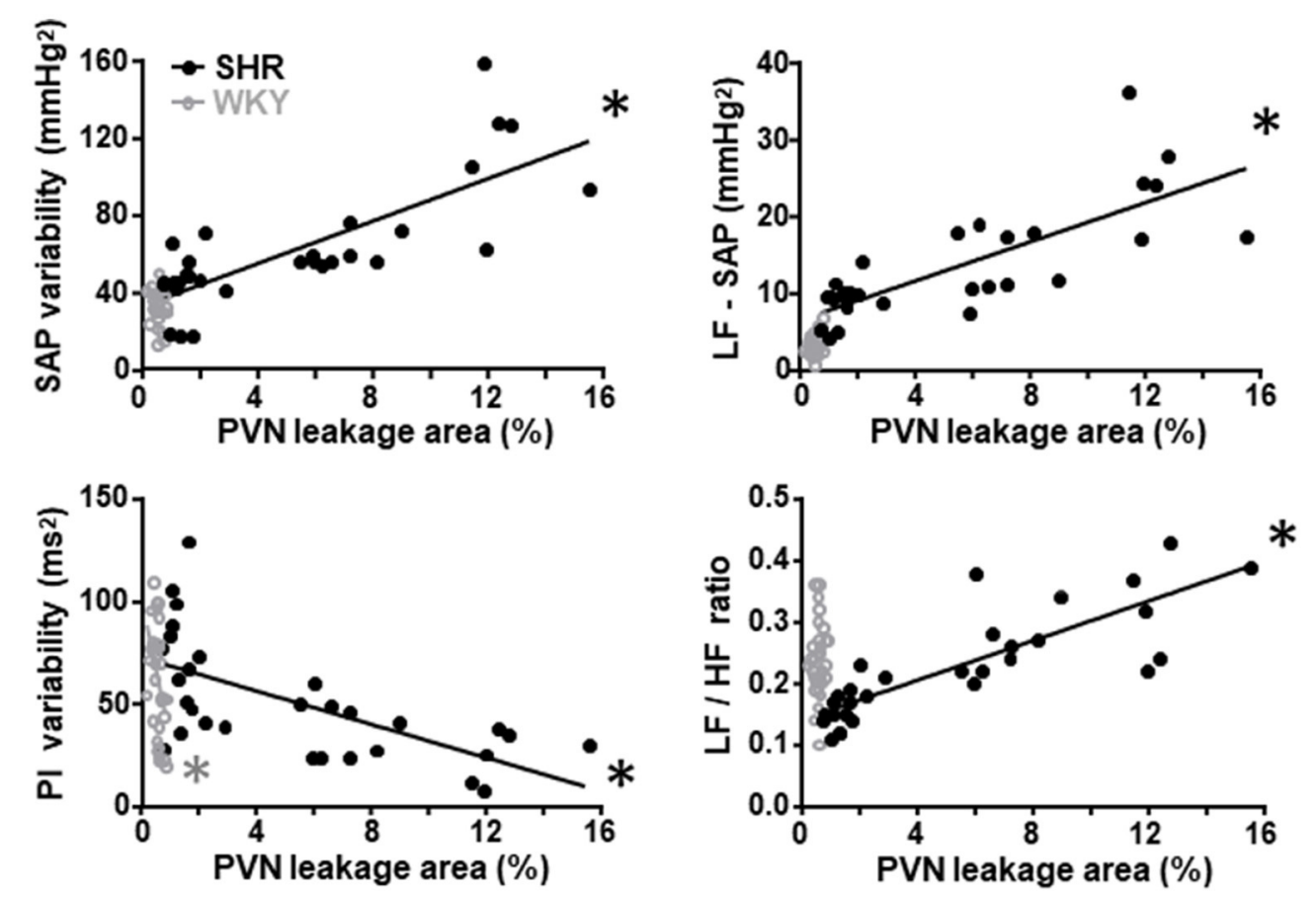

FIGURE 3 | Correlations between PVN blood-brain barrier permeability and autonomic parameters in sedentary and trained spontaneously hypertensive (SHR) and normotensive rats (WKY). In the SHR group, training-induced reduction in the leakage area was positively correlated with reduced systolic arterial pressure (SAP) variability, with its low frequency component (LF) and with decreased LF/HF ratio to the heart. Reduced leakage area was negatively correlated with increased pulse interval $(\mathrm{PI})$ variability. Correlation coefficients and $P$-values for regression equations are presented in Table 4. ${ }^{*}$ denotes a significant correlation.

availability (with decreased microglia activation) contributes to the improvement of BBB integrity in the trained SHR since simultaneous Ang II infusion blocks these responses; (v) Ang II blockade of training effects on BBB permeability is a prompt response in the PVN with minor effects in other autonomic areas after 14-days stimulation; (vi) in the WKY group repetitive exercise also induces a mild reduction of BBB permeability within the PVN and NTS, effects significantly correlated with the establishment of resting bradycardia in the normotensive strain.

The BBB, a complex multicellular structure acting as a selective barrier between the systemic circulation and the central nervous system, restricts the entry of substances into the brain by the transcellular (mediated by specific transporters and receptor proteins across the endothelial cell) and the paracellular transport (high electrical resistance tight junctions). Several techniques (dyes and/or radioactive substances' permeability, transendothelial electrical resistance, immunohistochemistry, RT-PCR, western blotting, electron microscopy) have been used to quantify $\mathrm{BBB}$ integrity/disruption and the expression of $\mathrm{BBB}$ constituents. Although $\mathrm{BBB}$ leakage induced by either hypertension, stroke, hyperosmolar stimulus, and aging has been reported in brain areas related to cognitive impairment since the 80's (Mueller and Heistad, 1980; Tamaki et al., 1984; Amenta et al., 2003; Ueno et al., 2004; Al-Sarraf et al., 2007; Zhang et al., 2010; Capone et al., 2011; Faraco and Iadecola, 2013; Iadecola, 2015), information on the conditioning mechanism(s) are scarce. Regarding $\mathrm{BBB}$ leakage in hypertension, there are conflicting evidence since studies reported loss of tight junction proteins within the cortex and hippocampus (Pelisch et al., 2011; Mohammadi and Dehghani, 2014; Zhang et al., 2015) as well as no evidence of ultrastructural tight junctions' disruption (Ueno et al., 2004). Absence of tight junction breakdown was also reported in rats submitted to focal cerebral ischemia (Krueger et al., 2013). Recent studies have shown that hypertension is accompanied by increased $\mathrm{BBB}$ permeability within the PVN, NTS, and RVLM, important autonomic areas controlling sympathetic and parasympathetic outflow to heart and blood vessels (Yao and May, 2013; Biancardi et al., 2014; Biancardi and Stern, 2016). Our data showing increased FITC leakage into the brain parenchyma of the three autonomic areas in adult SHR-S confirmed these previous reports showing in addition that an intact $\mathrm{BBB}$ was present in the pre-hypertensive phase. Although we did not measure brain Ang II availability during the establishment of hypertension, it is highly possible that local Ang II (and its downstream pathways) activated by pressure elevation, participate in BBB extravasation. Indeed, we showed previously in chronically hypertensive aortic coarcted rats a marked activation of brain RAS, as confirmed by augmented angiotensinogen and AT1 receptor expression within the NTS and by the blockade of both, accompanied by pressure reduction after losartan treatment (Sangaleti et al., 2004). Another original observation arising from our data was that, different from other autonomic areas, $\mathrm{PVN}$ BBB leakage is progressive during the chronic phase of hypertension, as observed in SHR-S from 3 to 5 
TABLE 4 | Correlation coefficients $(r)$ and $P$-values of regression equations correlating training-induced changes on autonomic control with training-induced changes on BBB leakage within the PVN, NTS, and RVLM of WKY and SHR groups.

\begin{tabular}{|c|c|c|}
\hline & \multicolumn{2}{|c|}{ Groups } \\
\hline & WKY & SHR \\
\hline \multicolumn{3}{|l|}{ PVN } \\
\hline BBB leakage $\times$ SAP variability & $r=-0.140 P=0.461$ & $r=0.778 P<0.001^{\#}$ \\
\hline BBB leakage x LF-SAP & $r=0.337 P=0.069$ & $r=0.779 P<0.001^{\#}$ \\
\hline BBB leakage $\times$ PI variability & $r=-0.421 P=0.021$ & $r=-0.632 P<0.001^{\#}$ \\
\hline BBB leakage x LF/HF ratio & $r=0.075 P=0.696$ & $r=0.827 P<0.001^{\#}$ \\
\hline BBB leakage $\times \alpha$ LF & $r=0.040 P=0.832$ & $r=-0.587 P<0.001^{\#}$ \\
\hline BBB leakage $\mathrm{x} \alpha \mathrm{HF}$ & $r=-0.312 P=0.094$ & $r=-0.417 P=0.022$ \\
\hline \multicolumn{3}{|l|}{ NTS } \\
\hline BBB leakage $\times$ SAP variability & $r=0.215 P=0.255$ & $r=0.744 P<0.001^{\#}$ \\
\hline BBB leakage $\times$ LF-SAP & $r=0.307 P=0.099$ & $r=0.732 P<0.001^{\#}$ \\
\hline BBB leakage $\times$ PI variability & $r=-0.130 P=0.495$ & $r=-0.686 P<0.001^{\#}$ \\
\hline BBB leakage $x$ LF/HF ratio & $r=-0.116 P=0.542$ & $r=0.686 P<0.001^{\#}$ \\
\hline BBB leakage $\times \alpha$ LF & $r=-0.151 P=0.427$ & $r=-0.656 P<0.001^{\#}$ \\
\hline BBB leakage $\mathrm{x} \alpha \mathrm{HF}$ & $r=-0.393 P=0.032$ & $r=-0.466 P=0.010$ \\
\hline \multicolumn{3}{|l|}{ RVLM } \\
\hline BBB leakage $\times$ SAP variability & $r=0.187 P=0.323$ & $r=0.605 P<0.001^{\#}$ \\
\hline BBB leakage $\times$ LF-SAP & $r=0.298 P=0.110$ & $r=0.731 P<0.001^{\#}$ \\
\hline BBB leakage x PI variability & $r=0.065 P=0.733$ & $r=-0.616 P<0.001^{\#}$ \\
\hline BBB leakage $\times$ LF/HF ratio & $r=0.061 P=0.749$ & $r=0.723 P<0.001^{\#}$ \\
\hline BBB leakage $\times \alpha$ LF & $r=0.354 P=0.055$ & $r=-0.525 P=0.003$ \\
\hline BBB leakage $\times \alpha \mathrm{HF}$ & $r=-0.099 P=0.601$ & $r=-0.404 P=0.027$ \\
\hline
\end{tabular}

SAP, Systolic arterial pressure; PI, pulse interval; LF, low-frequency component; $H F$, high-frequency component; $\alpha L F$ and $\alpha H F$, spontaneous baroreflex sensitivity estimated by LF and HF spectral components respectively. Correlations include 24-30 values of sedentary and trained rats ( $n=4$ rats/subgroup) from all time points. "denotes a significant correlation after Bonferroni correction of the significant level $\left(\alpha_{c}=0.0028\right)$ for the 18 analysis made.

months. Additionally, we showed that Ang II effect on BBB lesion was more prominent within the PVN, since changes in leakage area and EBA expression following icv Ang II were higher and faster than those observed in the NTS and RVLM. Moreover, our data on the absence of FITC leakage in the hypoglossus nucleus reinforces previous observation on the lack of BBB disruption in non-cardiovascular related areas (Biancardi et al., 2014). Therefore, it appears that hypertension damages preferentially the microcirculatory profile within brain autonomic nuclei (as previously described, Yao and May, 2013; Biancardi et al., 2014; Biancardi and Stern, 2016) as well as in hippocampal and cortical areas (Ueno et al., 2009; Pelisch et al., 2013; Mohammadi and Dehghani, 2014; Takeuchi et al., 2015).

A very important observation of the present study was the rapid correction of $\mathrm{BBB}$ leakage in autonomic areas of trained SHR, which was normalized after 2 weeks of exercise training. Importantly, the early restoration of BBB integrity in the PVN, NTS, and RVLM of trained SHR occurred simultaneously with the improvement of autonomic control and preceded the significant AP and HR reduction only observed after the 4th week of training. The improvement of autonomic control was confirmed by the decrease of both $\mathrm{LF} / \mathrm{HF}$ ratio in the heart (due to a marked HF increase, indicative of parasympathetic modulation) and sympathetic vasomotor variability. These responses improved PI variability and abrogated the continuous increase in SAP variability, characteristics of the sedentary SHR. Moreover, strong positive correlations were observed between BBB leakage in the three autonomic areas and SAP variability, LF-SAP, and LF/HF ratio, while PI variability was negatively correlated with BBB leakage. Previous studies have already documented the efficacy of training to improve autonomic control of the circulation in hypertension (Pan et al., 2007; Agarwal et al., 2011; Masson et al., 2014, 2015) and other cardiovascular diseases (Gao et al., 2007; Ichige et al., 2016), but to our knowledge this is the first report showing that traininginduced preservation of BBB integrity correlates with improved autonomic control in hypertensive individuals.

We did not measure Ang II content changes in autonomic areas, but we proved its role in training-induced improvement on BBB function since simultaneous icv Ang II infusion completely blocked training effects on both FITC leakage and microglia expression within the PVN. This seems to be a local Ang II effect because a very low, subpressor dose (12.5 ng/h) was infused into the lateral ventricle. The present observations confirmed our previous findings in SHR submitted to a similar $\mathrm{T}$ protocol that training downregulates RAS within the PVN, NTS, and RVLM (Chaar et al., 2015) showing in addition that microglia inactivation could contribute to the prevention of BBB dysfunction in SHR-T. Previous studies have already demonstrated the relationship between increased brain Ang II availability, augmented reactive oxygen species and proinflammatory cytokines, microglial activation and loss of BBB integrity (Shi et al., 2010; Zubcevic et al., 2011; Biancardi et al., 2014). The new finding of the present study is that training, by reducing Ang II availability, is able to interrupt this vicious cycle and preserve $\mathrm{BBB}$ integrity even in the presence of hypertension. Additional support for this proposition is our observations on both $\mathrm{AT}_{1}$ receptors' downregulation following exogenous Ang II infusion and training-induced $\mathrm{AT}_{1}$ receptors' upregulation, which is suggestive of reduced Ang II availability within autonomic areas of the SHR-T. Indeed, in age-matched SHR submitted to a similar training protocol, we have already shown a prompt (2 weeks) downregulation of the RAS within the PVN, NTS, and RVLM simultaneously with reduced sympathetic and increased parasympathetic activity to the heart, decreased vasomotor sympathetic outflow and smaller pressure variability (Felix and Michelini, 2007; Chaar et al., 2015). In trained adult SHR we also showed normalization of autonomic dysfunction and baroreflex sensitivity occurring simultaneously with an early ( 2 weeks) and marked decrease of $\mathrm{p} 47^{\text {phox }}$ and $\mathrm{gp} 91^{\text {phox }}$ expression, reduced oxidative stress, decreased phosphorylation of ERK1/2, normalization of NF-kB translocation to the nucleus, reduced microglia activation and decreased inflammation within the PVN (Masson et al., 2014, 2015). These observations reinforce the deactivation of Ang II and its downstream pathways by the exercise training. Consistent with those findings, our data on augmented EBA expression within the PVN, NTS, and RVLM confirmed the training-induced improvement of BBB 


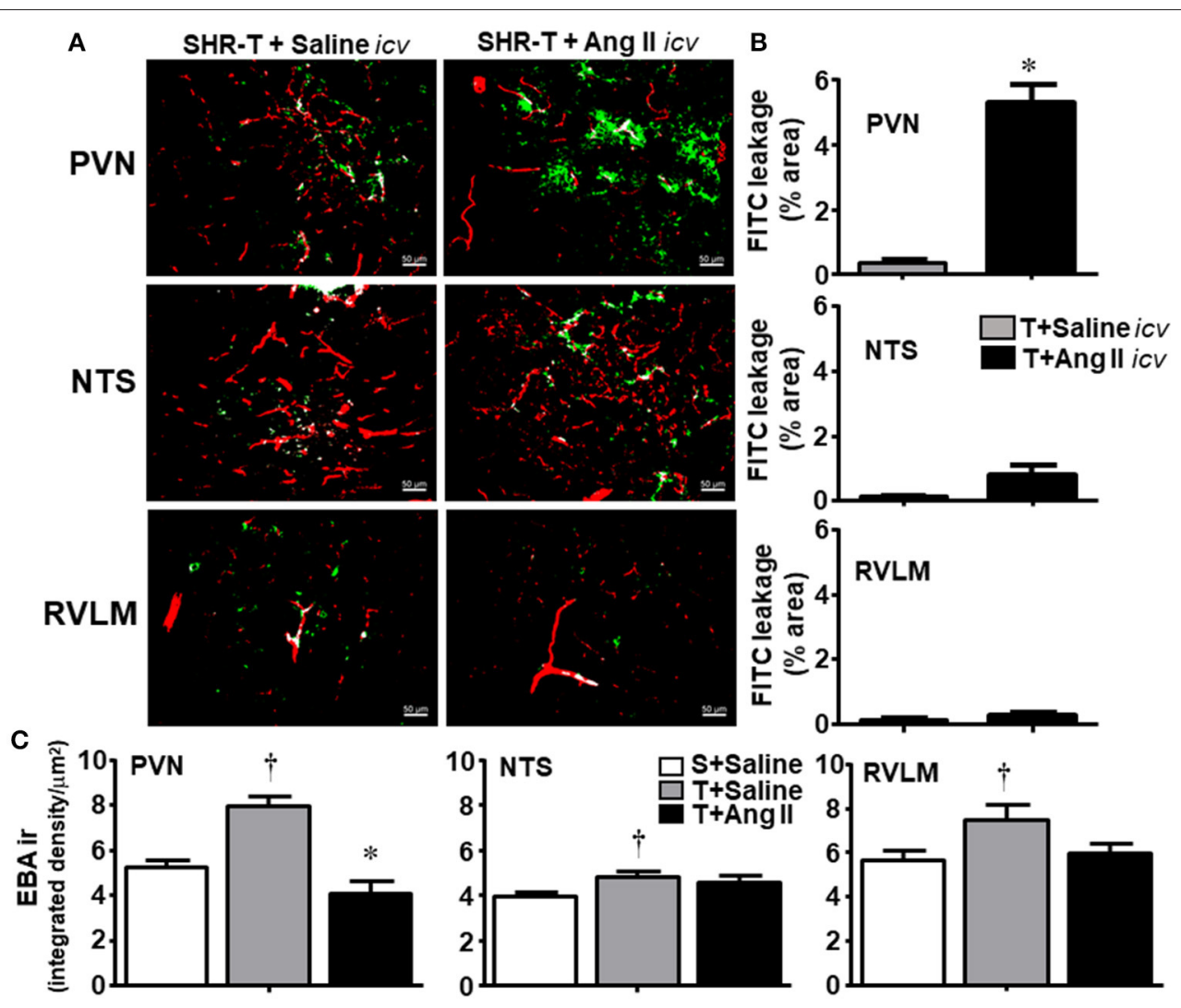

FIGURE 4 | Combined effect of aerobic training (T) and angiotensin II (Ang II) infusion on blood-brain barrier permeability in autonomic areas of spontaneously hypertensive rats (SHR) aged 3 months. (A) Representative images showing capillary profile (red) and FITC extravasation (green) within the brain parenchyma of the paraventricular nucleus of the hypothalamus (PVN), nucleus of the solitary tract (NTS) and rostral ventrolateral medulla (RVLM) in SHR-T infused with saline or Ang II icv. (B). Quantification of the leakage area in the three autonomic nuclei of SHR-T infused with saline or Ang II icv. Measurements were made in 7-9 slices, 3-4 rats/group. Significance $(P<0.05){ }^{*}$ vs. saline. (C) Effects of T and T associated with Ang II icv infusion on the expression of endothelial barrier antigen (EBA ir) within the PVN, NTS, and RVLM. EBA ir in the sedentary (S) SHR infused with saline is shown for comparison. Significances $(P<0.05)$ are ${ }^{\dagger}$ vs. S+saline; ${ }^{*}$ vs. $T+$ saline. $^{2}$

integrity after 2 weeks of exercise, while their reduction in SHRT simultaneously infused with Ang II validated the involvement of local RAS on BBB effects induced by training. The efficacy of training to improve $\mathrm{BBB}$ integrity and ameliorate brain functions was also documented in other pathologies as diabetes, stroke and neurodegenerative diseases (Ding et al., 2006; Zhang et al., 2013; Wang et al., 2014; de Senna et al., 2015; Souza et al., 2016). In addition, trained normotensive rats exhibited small improvements on both BBB integrity and PI variability, reinforcing the importance of an almost intact $\mathrm{BBB}$ for normal brain perfusion and adequate neuronal activity that drives the autonomic control of the circulation.

We are aware that commercial AT1 receptor antibodies are non-specific, which could represent a caveat in the interpretation of its immunohistochemistry data. For this reason, we confirmed the involvement of local RAS by comparing BBB permeability in trained SHR simultaneously infused with saline or Ang II icv. These functional data do support the role played by the vasoconstrictor, proliferative, pro-inflammatory axis of the brain RAS in damaging (high Ang II content in the SHRS) or improving (low Ang II availability in SHR-T) the BBB permeability. We also identified training-induced time course and the magnitude of BBB changes within the PVN, NTS, and RVLM, but we did not clarify whether reduced BBB extravasation in exercised SHR was due to changes in paracellular and/or transcellular transport across the endothelium. In addition, our experiments did not reveal the barrier component(s) contributing to hypertension-induced deficits and traininginduced benefits observed in sedentary and trained SHR, respectively. Experiments are now being conducted in our laboratory in order to elucidate the mechanism(s) by which aerobic training reverses and corrects $\mathrm{BBB}$ permeability in hypertensive individuals. Nevertheless, our data disclose the importance of training to change tissue Ang II content (and microglia activation), whose reduction is crucial for both the maintenance of $\mathrm{BBB}$ integrity and the normalization of autonomic control of the circulation in hypertensive individuals.

In conclusion the present set of data uncover a novel benefit of exercise training for hypertensive individuals. This non-pharmacological tool proved to be highly efficient in reversing the deleterious effect of hypertension on BBB leakage within autonomic areas, an effect strongly correlated with 


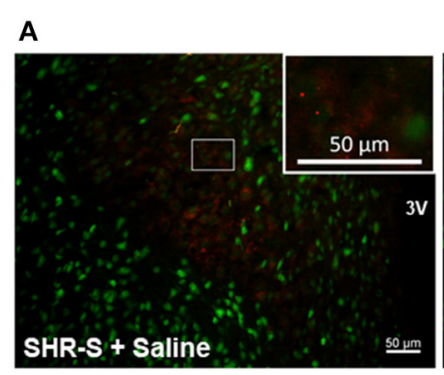

PVN (Neu-N - AT1 receptor)

B
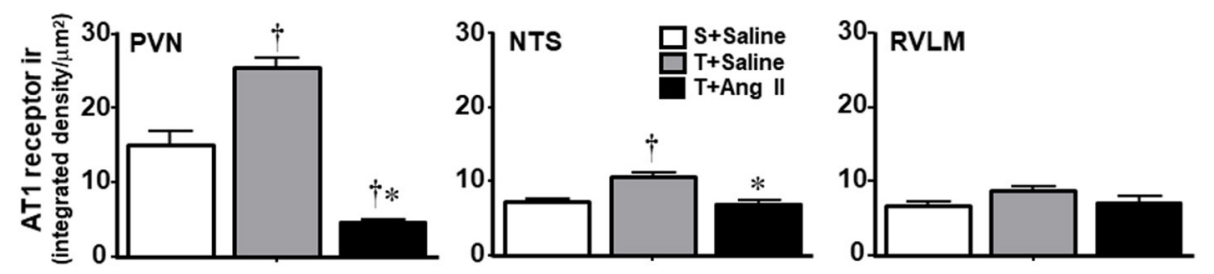

FIGURE 5 | Combined effect of aerobic training (T) and angiotensin II (Ang II) infusion on AT1 receptor expression in autonomic nuclei of spontaneously hypertensive rats (SHR) aged 3 months. (A) Representative images comparing AT1 receptor expression (red) within the paraventricular nucleus of the hypothalamus (PVN) in SHR-T infused with saline or angiotensin II (Ang II). Neurons are identified by Neu-N staining (green). Insets are high magnification of the white rectangle depicting representative neurons that show increased receptors' expression in SHR-T+Saline and decreased in SHR-T+Ang II when compared to SHR-S+saline.

(B) Quantification of AT1 receptor density within the PVN, nucleus of the solitary tract (NTS) and rostral ventrolateral medulla (RVLM) in SHR-T infused with saline or Ang II icv. AT1 receptor expression in the sedentary (S) SHR infused with saline is also shown for comparison. Measurements were made in 7-9 slices, 3-4 rats/group. Significances $(P<0.05)$ are ${ }^{\dagger}$ vs. S+saline; *vs. T+ saline.

\section{A}

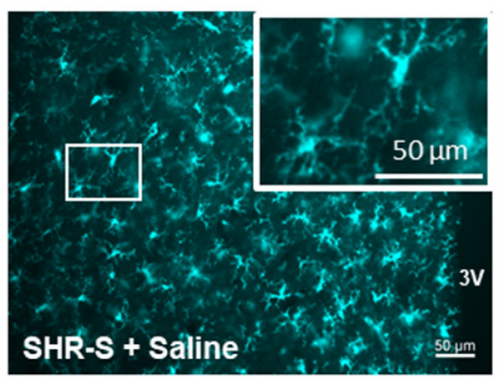

PVN: IBA 1
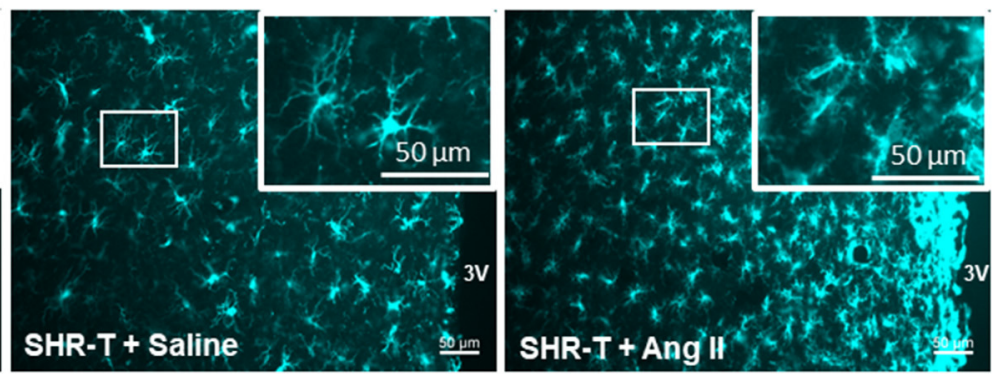

B
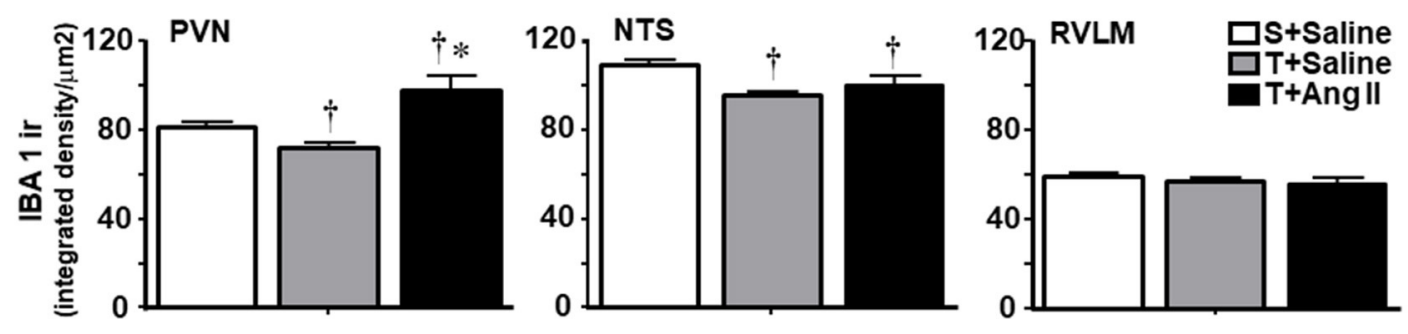

FIGURE 6 | Combined effect of aerobic training (T) and angiotensin II (Ang II) infusion on microglia expression (IBA1 ir) in autonomic nuclei of spontaneously hypertensive rats (SHR) aged 3 months. (A) Representative images comparing IBA1 expression (blue) within the paraventricular nucleus of the hypothalamus (PVN) in SHR-T infused with saline or angiotensin II (Ang II). Insets depict high magnification of areas delimited by the white rectangle and show both the maintenance of inactivated microglia in SHR-T+Saline and activated pattern in SHR-T+Ang II. (B) Quantification of IBA1 density in the PVN, nucleus of tract solitary (NTS) and rostral ventrolateral medulla (RVLM) in SHR-T infused with saline or Ang II icv. IBA1 expression in the sedentary (S) SHR infused with saline is also shown for comparison. Measurements were made in 7-9 slices, 3-4 rats/group. Significances $(P<0.05)$ are ${ }^{\dagger}$ vs. S+saline; *vs. T+ saline. 
the improvement of both parasympathetic and sympathetic control of cardiovascular parameters, even in the persistence of hypertension. Since aerobic training has no side effects, it is a useful adjuvant therapeutic strategy to maintain BBB integrity and adequate tissue perfusion, with clear clinical implications.

\section{AUTHOR CONTRIBUTIONS}

LB: participated in the research design, acquired and interpreted data, wrote the manuscript; MJ: participated in the research design, acquired and interpreted data; $\mathrm{MF}, \mathrm{AR}$, and $\mathrm{AC}$ : performed the experiments, analyzed, and revised data; LM:

\section{REFERENCES}

Agarwal, D., Welsch, M. A., Keller, J. N., and Francis, J. (2011). Chronic exercise modulates RAS components and improves balance between pro-and antiinflammatory cytokines in the brain of SHR. Basic Res. Cardiol. 106, 1069-1085. doi: 10.1007/s00395-011-0231-7

Al-Sarraf, H., Ghaaedi, F., and Redzic, Z. (2007). Time course of hyperosmolar opening of the blood-brain and blood-CSF barriers in spontaneously hypertensive rats. J. Vasc. Res. 44, 99-109. doi: 10.1159/000098260

Amenta, F., Di Tullio, M. A., and Tomassoni, D. (2003). Arterial hypertension and brain damage-evidence from animal models (review). Clin. Exp. Hypertens. 25, 359-380. doi: 10.1081/CEH-120023545

Biancardi, V. C., and Stern, J. E. (2016). Compromised blood-brain barrier permeability: novel mechanism by which circulating angiotensin II signals to sympathoexcitatory centres during hypertension. J. Physiol. 594, 1591-1600. doi: 10.1113/JP271584

Biancardi, V. C., Son, S. J., Ahmadi, S., Filosa, J. A., and Stern, J. E. (2014). Circulating angiotensin II gains access to the hypothalamus and brain stem during hypertension via breakdown of the blood-brain barrier. Hypertension 63, 572-579. doi: 10.1161/HYPERTENSIONAHA.113.01743

Biancardi, V. C., Stranahan, A. M., Krause, E. G., de Kloet, A. D., and Stern, J. E. (2016). Cross talk between AT1 receptors and Toll-like receptor 4 in microglia contributes to angiotensin II-derived ROS production in the hypothalamic paraventricular nucleus. Am. J. Physiol. Heart Circ. Physiol. 310, H404-H415. doi: 10.1152/ajpheart.00247.2015

Capone, C., Faraco, G., Park, L., Cao, X., Davisson, R. L., and Iadecola, C. (2011). The cerebrovascular dysfunction induced by slow pressor doses of angiotensin II precedes the development of hypertension. Am. J. Physiol. Heart Circ. Physiol. 300, H397-H407. doi: 10.1152/ajpheart.00679.2010

Cavalleri, M. T., Burgi, K., Cruz, J. C., Jordão, M. T., Ceroni, A., and Michelini, L. C. (2011). Afferent signaling drives oxytocinergic preautonomic neurons and mediates training-induced plasticity. Am. J. Physiol. Regul. Integr. Comp. Physiol. 301, R958-R966. doi: 10.1152/ajpregu.00104.2011

Ceroni, A., Chaar, L. J., Bombein, R. L., and Michelini, L. C. (2009). Chronic absence of baroreceptor inputs prevents training-induced cardiovascular adjustments in normotensive and spontaneously hypertensive rats. Exp. Physiol. 94, 630-640. doi: 10.1113/expphysiol.2008.046128

Chaar, L. J., Alves, T. P., Batista Junior, A. M., and Michelini, L. C. (2015). Early training-induced reduction of angiotensinogen in autonomic areas-the main effect of exercise on brain renin-angiotensin system in hypertensive rats. PLoS ONE 10:e0137395. doi: 10.1371/journal.pone.0137395

de Senna, P. N., Xavier, L. L., Bagatini, P. B., Saur, L., Galland, F., Zanotto, C., et al. (2015). Physical training improves non-spatial memory, locomotor skills and the blood brain barrier in diabetic rats. Brain Res. 1618, 75-82. doi: 10.1016/j.brainres.2015.05.026

de Vries, H. E., Kuiper, J., de Boer, A. G., Van Berkel, T. J., and Breimer, D. D. (1997). The blood-brain barrier in neuroinflammatory diseases. Pharmacol. Rev. 49, 143-155.

DeVos, S. L., and Miller, T. M. (2013). Direct intraventricular delivery of drugs to the rodent central nervous system. J. Vis. Exp.12:e50326. doi: 10.3791/50326 conceived, designed and coordinated the research, wrote and revised the manuscript; All authors read and approved the final draft of the manuscript.

\section{FUNDING}

This study was supported by Fundação de Amparo à Pesquisa do Estado de São Paulo [FAPESP, grants number 11/14395-1 (fellowship to LB), 11/51410-9 and 15/24935-4 (research grants to LM)] and Conselho Nacional de Desenvolvimento Científico e Tecnológico [CNPq, grants 304060/2011-9 and 305940/2015-5, research fellow to LM].

Ding, Y. H., Ding, Y., Li, J., Bessert, D. A., and Rafols, J. A. (2006). Exercise preconditioning strengthens brain microvascular integrity in a rat stroke model. Neurol. Res. 28, 184-189. doi: 10.1179/016164106X98053

Eckberg, D. L. (1997). Sympathovagal balance: a critical appraisal. Circulation 96, 3224-3232. doi: 10.1161/01.CIR.96.9.3224

Erickson, M. A., and Banks, W. A. (2013). Blood-brain barrier dysfunction as a cause and consequence of Alzheimer's disease. J. Cereb. Blood Flow Metab. 33, 1500-1513. doi: $10.1038 /$ jcbfm.2013.135

Faraco, G., and Iadecola, C. (2013). Hypertension: a harbinger of stroke and dementia. Hypertension 62, 810-817. doi: 10.1161/HYPERTENSIONAHA.113.01063

Felix, J. V., and Michelini, L. C. (2007). Training-induced pressure fall in spontaneously hypertensive rats is associated with reduced angiotensinogen mRNA expression within the nucleus tractus solitarii. Hypertension 50, 780-785. doi: 10.1161/HYPERTENSIONAHA.107.094474

Gao, L., Wang, W., Liu, D., and Zucker, I. H. (2007). Exercise training normalizes sympathetic outflow by central antioxidant mechanisms in rabbits with pacing-induced chronic heart failure. Circulation 115, 3095-3102. doi: 10.1161/CIRCULATIONAHA.106.677989

Hagan, N., and Ben-Zvi, A. (2015). The molecular, cellular, and morphological components of blood-brain barrier development during embryogenesis. Semin. Cell Dev. Biol. 38, 7-15. doi: 10.1016/j.semcdb.2014.12.006

Higa-Taniguchi, K. T., Silva, F. C., Silva, H. M., Michelini, L. C., and Stern, J. E. (2007). Exercise training-induced remodeling of paraventricular nucleus (nor)adrenergic innervation in normotensive and hypertensive rats. Am. J. Physiol. Regul. Integr. Comp. Physiol. 292, R1717-R1727. doi: 10.1152/ajpregu.00613.2006

Iadecola, C. (2015). Dangerous leaks: blood-brain barrier woes in the aging hippocampus. Neuron 85, 231-233. doi: 10.1016/j.neuron.2014.12.056

Ichige, M. H., Santos, C. R., Jordão, C. P., Ceroni, A., Negrão, C. E., and Michelini, L. C. (2016). Exercise training preserves vagal preganglionic neurones and restores parasympathetic tonus in heart failure. J. Physiol. 594, 6241-6254. doi: 10.1113/JP272730

James, P. A., Oparil, S., Carter, B. L., Cushman, W. C., Dennison-Himmelfarb, C., Handler, J., et al. (2014). 2014 Evidence-Based guideline for the management of high blood pressure in adults: report from the panel members appointed to the eighth joint national committee (JNC 8). JAMA 311, 507-520. doi: 10.1001/jama.2013.284427

Japundzic, N., Grichois, M. L., Zitoun, P., Laude, D., and Elghozi, J. L. (1990). Spectral analysis of blood pressure and heart rate in conscious rats: effects of autonomic blockers. J. Auton. Nerv. Syst. 30, 91-100. doi: 10.1016/0165-1838(90)90132-3

Kang, Y. M., Ma, Y., Zheng, J. P., Elks, C., Sriramula, S., Yang, Z. M., et al. (2009). Brain nuclear factor-kappa B activation contributes to neurohumoral excitation in angiotensin II-induced hypertension. Cardiovasc. Res. 82, 503-512. doi: 10.1093/cvr/cvp073

Krueger, M., Härtig, W., Reichenbach, A., Bechmann, I., and Michalski, D. (2013). Blood-brain barrier breakdown after embolic stroke in rats occurs without ultrastructural evidence for disrupting tight junctions. PLOS ONE 8:e56419. doi: 10.1371/journal.pone.0056419 
Kucuk, M., Kaya, M., Kalayci, R., Cimen, V., Kudat, H., Arican, N., et al. (2002). Effects of losartan on the blood-brain barrier permeability in longterm nitric oxide blockade-induced hypertensive rats. Life Sci. 71, 937-946. doi: 10.1016/S0024-3205(02)01772-1

Laude, D., Elghozi, J. L., Girard, A., Bellard, E., Bouhaddi, M., Castiglioni, P., et al. (2004). Comparison of various techniques used to estimate spontaneous baroreflex sensitivity (the EuroBaVar study). Am. J. Physiol. 286, R226-R231. doi: 10.1152/ajpregu.00709.2002

Lin, B., and Ginsberg, M. D. (2000). Quantitative assessment of the normal cerebral microvasculature by endothelial barrier antigen (EBA) immunohistochemistry: application to focal cerebral ischemia. Brain Res. 865, 237-244. doi: 10.1016/S0006-8993(00)02228-9

Mamo, J. C. L., Lam, V., Giles, C., Coulson, S. H., Fimognari, N., Mooranian, A., et al. (2017). Antihypertensive agents do not prevent blood-brain barrier dysfunction and cognitive deficits in dietary-induced obese mice. Int. J. Obes. 41, 926-934. doi: 10.1038/ijo.2017.57

Martelli, D., Silvani, A., McAllen, R. M., May, C. N., and Ramchandra, R. (2014). The low frequency power of heart rate variability is neither a measure of cardiac sympathetic tone nor of baroreflex sensitivity. Am. J. Physiol. Heart Circ. Physiol. 307, H1005-H1012. doi: 10.1152/ajpheart.00361.2014

Masson, G. S., Costa, T. S., Yshii, L., Fernandes, D. C., Soares, P. P., Laurindo, F. R., et al. (2014). Time-dependent effects of training on cardiovascular control in spontaneously hypertensive rats: role for brain oxidative stress and inflammation and baroreflex sensitivity. PLoS ONE 9:e94927. doi: 10.1371/journal.pone.0094927

Masson, G. S., Nair, A. R., Silva Soares, P. P., Michelini, L. C., and Francis, J. (2015). Aerobic training normalizes autonomic dysfunction, HMGB1 content, microglia activation and inflammation in hypothalamic paraventricular nucleus of SHR. Am. J. Physiol. Heart Circ. Physiol. 309, H1115-H1122. doi: 10.1152/ajpheart.00349.2015

Mohammadi, M. T., and Dehghani, G. A. (2014). Acute hypertension induces brain injury and blood-brain barrier disruption through reduction of claudins mRNA expression in rat. Pathol. Res. Pract. 210, 985-990. doi: 10.1016/j.prp.2014.05.007

Mueller, S. M., and Heistad, D. D. (1980). Effect of chronic hypertension on the blood-brain barrier. Hypertension 2, 809-812. doi: 10.1161/01.HYP.2.6.809

Norsted, E., Gömüç, B., and Meister, B. (2008). Protein components of the bloodbrain barrier (BBB) in the mediobasal hypothalamus. J. Chem. Neuroanat. 36, 107-121. doi: 10.1016/j.jchemneu.2008.06.002

Pan, Y. X., Gao, L., Wang, W. Z., Zheng, H., Liu, D., Patel, K. P., et al. (2007). Exercise training prevents arterial baroreflex dysfunction in rats treated with central angiotensin II. Hypertension 49, 519-527. doi: 10.1161/01.HYP.0000256955.74461.93

Paxinos, G., and Watson, C. (2007). The Rat Brain in Stereotaxic Coordinates, 6 th $E d n$. San Diego, CA: Academic Press.

Pelisch, N., Hosomi, N., Mori, H., Masaki, T., and Nishiyama, A. (2013). RAS inhibition attenuates cognitive impairment by reducing blood- brain barrier permeability in hypertensive subjects. Curr. Hypertens. Rev. 9, 93-98. doi: 10.2174/15734021113099990003

Pelisch, N., Hosomi, N., Ueno, M., Nakano, D., Hitomi, H., Mogi, M., et al. (2011). Blockade of AT1 receptors protects the blood-brain barrier and improves cognition in Dahl salt-sensitive hypertensive rats. Am. J. Hypertens. 24, 362-368. doi: 10.1038/ajh.2010.241

Rosenberg, G. A. (2012). Neurological diseases in relation to the blood-brain barrier. J. Cereb. Blood Flow Metab. 32, 1139-1151. doi: 10.1038/jcbfm.2011.197

Sangaleti, C. T., Crescenzi, A., and Michelini, L. C. (2004). Endogenous angiotensin and pressure modulate brain angiotensinogen and AT1A mRNA expression. Hypertension 43, 317-323. doi: 10.1161/01.HYP.0000112030.79692.21

Schoknecht, K., David, Y., and Heinemann, U. (2015). The blood-brain barriergatekeeper to neuronal homeostasis: clinical implications in the setting of stroke. Semin. Cell Dev. Biol. 38, 35-42. doi: 10.1016/j.semcdb.2014.10.004

Shi, P., Diez-Freire, C., Jun, J. Y., Qi, Y., Katovich, M. J., Li, Q., et al. (2010). Brain microglial cytokines in neurogenic hypertension. Hypertension 56, 297-303. doi: 10.1161/HYPERTENSIONAHA.110.150409
Souza, P. S., Gonçalves, E. D., Pedroso, G. S., Farias, H. R., Junqueira, S. C., Marcon, R., et al. (2016). Physical exercise attenuates experimental autoimmune encephalomyelitis by inhibiting peripheral immune response and blood-brain barrier disruption. Mol. Neurobiol. 54, 4723-4737. doi: 10.1007/s12035-016-0014-0

Takeuchi, S., Nagatani, K., Otani, N., Nawashiro, H., Sugawara, T., Wada, K., et al. (2015). Hydrogen improves neurological function through attenuation of blood-brain barrier disruption in spontaneously hypertensive stroke-prone rats. BMC Neurosci. 16:22. doi: 10.1186/s12868-015-0165-3

Tamaki, K., Sadoshima, S., and Heistad, D. D. (1984). Increased susceptibility to osmotic disruption of the blood-brain barrier in chronic hypertension. Hypertension 6, 633-638. doi: 10.1161/01.HYP.6.5.633

Task Force of the European Society of Cardiology, North American Society of Pacing and Electrophysiology. (1996). Heart rate variability standards of measurements, physiological interpretation and clinical use. Eur. Heart J. 17, 354-381. doi: 10.1093/oxfordjournals.eurheartj.a014868

Ueno, M., Nakagawa, T., Huang, C., Ueki, M., Kusaka, T., Hosomi, N., et al. (2009). The expression of P-glycoprotein is increased in vessels with bloodbrain barrier impairment in a stroke-prone hypertensive model. Neuropathol. Appl. Neurobiol. 35, 147-155. doi: 10.1111/j.1365-2990.2008.00966.x

Ueno, M., Sakamoto, H., Tomimoto, H., Akiguchi, I., Onodera, M., Huang, C. L., et al. (2004). Blood-brain barrier is impaired in the hippocampus of young adult spontaneously hypertensive rats. Acta Neuropathol. 107, 532-538. doi: 10.1007/s00401-004-0845-z

Waki, H., Gouraud, S. S., Maeda, M., Raizada, M. K., and Paton, J. F. (2011). Contributions of vascular inflammation in the brainstem for neurogenic hypertension. Respir. Physiol. Neurobiol. 178, 422-428. doi: 10.1016/j.resp.2011.05.004

Wang, X., Zhang, M., Feng, R., Li, W. B., Ren, S. Q., Zhang, J., et al. (2014). Physical exercise training and neurovascular unit in ischemic stroke. Neuroscience 271, 99-107. doi: 10.1016/j.neuroscience.2014.04.030

Yao, S. T., and May, C. N. (2013). Intra-carotid angiotensin II activates tyrosine hydroxylase-expressing rostral ventrolateral medulla neurons following blood-brain barrier disruption in rats. Neuroscience 245, 148-156. doi: 10.1016/j.neuroscience.2013.04.023

Zhang, M., Mao, Y., Ramirez, S. H., Tuma, R. F., and Chabrashvili, T. (2010). Angiotensin II induced cerebral microvascular inflammation and increased blood-brain barrier permeability via oxidative stress. Neuroscience 171, 852-858. doi: 10.1016/j.neuroscience.2010.09.029

Zhang, T., Su, J., Guo, B., Wang, K., Li, X., and Liang, G. (2015). Apigenin protects blood-brain barrier and ameliorates early brain injury by inhibiting TLR4-mediated inflammatory pathway in subarachnoid hemorrhage rats. Int. Immunopharmacol. 28, 79-87. doi: 10.1016/j.intimp.2015. 05.024

Zhang, Y., Zhang, P., Shen, X., Tian, S., Wu, Y., Zhu, Y., et al. (2013). Early exercise protects the blood-brain barrier from ischemic brain injury via the regulation of MMP-9 and Occludin in Rats. Int. J. Mol. Sci. 14, 11096-11112. doi: 10.3390/ijms140611096

Zubcevic, J., Waki, H., Raizada, M. K., and Paton, J. F. (2011). Autonomic-immune-vascular interaction: an emerging concept for neurogenic hypertension. Hypertension 57, 1026-1033. doi: 10.1161/ HYPERTENSIONAHA.111.169748

Conflict of Interest Statement: The authors declare that the research was conducted in the absence of any commercial or financial relationships that could be construed as a potential conflict of interest.

Copyright (c) 2017 Buttler, Jordão, Fragas, Ruggeri, Ceroni and Michelini. This is an open-access article distributed under the terms of the Creative Commons Attribution License (CC BY). The use, distribution or reproduction in other forums is permitted, provided the original author(s) or licensor are credited and that the original publication in this journal is cited, in accordance with accepted academic practice. No use, distribution or reproduction is permitted which does not comply with these terms. 